\title{
Interaction of nucleic acids with carbon nanotubes and dendrimers
}

\author{
Bidisha Nandy, Mogurampelly Santosh and Prabal K Maiti* \\ Centre for Condensed Matter Theory, Department of Physics, Indian Institute of Science, Bangalore 560 012, India \\ *Corresponding author (Fax, +91-80-23602602; Email, maiti@physics.iisc.ernet.in)
}

\begin{abstract}
Nucleic acid interaction with nanoscale objects like carbon nanotubes (CNTs) and dendrimers is of fundamental interest because of their potential application in CNT separation, gene therapy and antisense therapy. Combining nucleic acids with CNTs and dendrimers also opens the door towards controllable self-assembly to generate various supra-molecular and nano-structures with desired morphologies. The interaction between these nanoscale objects also serve as a model system for studying DNA compaction, which is a fundamental process in chromatin organization. By using fully atomistic simulations, here we report various aspects of the interactions and binding modes of DNA and small interfering RNA (siRNA) with CNTs, graphene and dendrimers. Our results give a microscopic picture and mechanism of the adsorption of single- and double-strand DNA (ssDNA and dsDNA) on CNT and graphene. The nucleic acid-CNT interaction is dominated by the dispersive van der Waals (vdW) interaction. In contrast, the complexation of DNA (both ssDNA and dsDNA) and siRNA with various generations of poly-amido-amine (PAMAM) dendrimers is governed by electrostatic interactions. Our results reveal that both the DNA and siRNA form stable complex with the PAMAM dendrimer at a physiological $\mathrm{pH}$ when the dendrimer is positively charged due to the protonation of the primary amines. The size and binding energy of the complex increase with increase in dendrimer generation. We also give a summary of the current status in these fields and discuss future prospects.
\end{abstract}

[Nandy B, Santosh M and Maiti PK 2012 Interaction of nucleic acids with carbon nanotubes and dendrimers. J. Biosci. 37 457-474] DOI 10.1007/ s12038-012-9220-8

\section{Introduction}

Nucleic acids (DNA and RNA) are the key components in many functions of the cell in living organisms and form the basis of life (Saenger 1984; Bloomfield et al. 2000; Rich and Zhang 2003). Nucleic acids undergo large structural transformations during many of the cell functioning operation such as transcription, translation and replication. These large structural transformations of nucleic acids are essentially triggered by protein binding or selective macromolecule binding to DNA. Double-stranded DNA (dsDNA) has many stable forms such as A-DNA, B-DNA and Z-DNA depending on the solvent condition and complexation with biomolecules such as proteins or enzymes. Thermodynamic stability of dsDNA in various such forms is essential for many biological functions (Jacobomolina et al. 1993; Kiefer et al. 1998; Jones et al. 1999; Lu et al. 2000). At physiological conditions, the B-form is favourable for dsDNA and A-form for RNA (Watson and Crick 1953; Wilkins et al.
1953; Franklin and Gosling 1953; Saenger 1984; Bloomfield et al. 2000; Klug 2004). However, for many biological functions, and for some biological organization, the Aform and Z-form of DNA are required (Saenger 1984; Bloomfield et al. 2000; Rich and Zhang 2003). Specific protein binding to the native B-DNA can trigger the transition from B- to A- or Z-DNA and stabilize the A-DNA and/ or Z-DNA form (Jacobomolina et al. 1993; Kiefer et al. 1998; Jones et al. 1999; Lu et al. 2000). ssDNA and dsDNA interactions with CNTs have been studied by several groups which have found promising applications in CNT separation and sensing within cells (Zheng et al. 2003a; Zheng et al. 2003b; Chou et al. 2004; Heller et al. 2006; Johnson et al. 2008; Zhao and Johnson 2007). However, it is not yet known whether CNTs or graphene can induce B- to A- or Z-DNA transition.

DNA compaction is one of the most important phenomenon in living organism where, DNA is stored in very compact form and some of its functions depend on the

Keywords. Binding and MD simulations; carbon nanotube; dendrimer; graphene; nucleic acids; van der Waals interaction 
degree of compaction. In some extreme cases, DNA is compacted a hundred times more than an unfolded form of DNA (Livolant 1991). One of the most well-known examples of DNA compaction is the individual nucleosome: a nucleosome protein complex with 147 base pairs of DNA is wrapped 1.65 times around a disk-shaped octamer of histone proteins (Schiessel 2003). The case of bacteriophage heads provides another example where DNA condensation is mediated by multi-cationic species, as reported in recent in vitro studies (Zinchenko and Chen 2006). Studies of DNA compaction by artificial nanoscale templates like synthetic dendrimers and nanotubes are inspired by the DNA-histone complex and are not yet well understood. When we deal with nanoscale templates, DNA condensation happens near the oppositely charged surface of the nanoscale object and the structural geometry of the nanoscale objects plays an important role in the mechanism of DNA compaction.

Another class of nucleic acid which is of great interest is the short interfering RNA (siRNA) or silencing RNA, which belongs to a class of double-stranded RNA (dsRNA). Eukaryotic cells use siRNA molecule to minimize the expression of pathogenic gene or overexpression of gene activity by the regulatory mechanism known as RNA interference (RNAi) (Napoli et al. 1990; Fire et al. 1998; Elbashir et al. 2001b; Elbashir et al. 2001a; Hannon 2002; Hannon and Rossi 2004; Meister and Tuschl 2004; Bergstrom and Antiat 2005). The discovery of RNAi has emerged as an attractive route where, inside a disease cell, affected gene can be silenced by delivering siRNA (Elbashir et al. 2001a; Soutschek et al. 2004; Kim and Rossi 2007). Similar to dsDNA, siRNA is also negatively charged and faces major barriers to enter through the cell membrane to the desired location during the delivery. Although siRNA is demonstrated to be potential silencers of gene expression that can have extraordinary treatment capabilities of HIV, hepatitis and cancer (Lee et al. 2002; Jacque et al. 2002; Zamore and Aronin 2003; Soutschek et al. 2004; Urban-Klein et al. 2005; Kim and Rossi 2007; Kurreck 2009), efficient delivery of these molecules to the target cell is a big challenge today. Another difficulty of siRNA-based therapy is the slow transfection through the membrane and siRNA degradation in serum. One of the potential solutions to overcome these problems is the compaction of siRNA by synthetic gene delivery systems (Mintzer and Simanek 2009). siRNA compaction can be accomplished by using a non-viral delivery carrier such as linear or branched cationic polymer (dendrimer) (Tsubouchi et al. 2002; Duxbury et al. 2003; Grayson et al. 2006) and CNTs (Lu et al. 2004; Liu et al. 2007, 2008, 2009).

In this article, we present some important findings on dsDNA stability and review a few recent theoretical studies from our group (Maiti and Bagchi 2006; Vasumathi and Maiti 2010; Nandy and Maiti 2011; Santosh et al. 2012) on DNA/siRNA interaction with CNTs and graphene as well as DNA/siRNA compaction by dendrimers. All the calculations reported were performed using atomistic molecular dynamics simulations in explicit solvent with neutralizing counterions.

\section{Stability of the dsDNA}

\subsection{A- to B-DNA transition in the presence of water and 'CNT+water'}

It is well known that at physiological conditions, the B-form is the most stable form of dsDNA and the A-form is the most stable form of RNA (Watson and Crick 1953; Wilkins et al. 1953; Franklin and Gosling 1953; Saenger 1984; Bloomfield et al. 2000; Klug 2004). The dsDNA can undergo a transition from B- to A-form by changing the solvent conditions or by binding to protein or by external force (Saenger 1984; Jacobomolina et al. 1993; Kiefer et al. 1998; Jones et al. 1999; Bloomfield et al. 2000; Lu et al. 2000; Zinchenko and Chen 2006; Santosh and Maiti 2009; Santosh and Maiti 2011). We studied the structural transition of dsDNA from A-form to B-form in the presence and absence of a CNT. This transition was well studied in 1990s by Kollman and co-workers, but the effect of the presence of a substrate like CNT on the transition has not been explored yet (Johnson et al. 2008). It is known that at physiological conditions in aqueous environment, A-DNA transforms into B-DNA in a few hundreds of picoseconds (Cheatham and Kollman 1996; Miller and Kollman 1997). We simulate the dsDNA starting from A-form in explicit solvent with neutralizing counterions to study the A-to-B transition. In another simulation setup, we included CNT with the initial A-form DNA to see the effect of CNT on the A-to-B transition. In our simulation of A-form DNA in water, we observed the transition of DNA from A-form to B-form within $600 \mathrm{ps}$. Various structural parameters that distinguish A-DNA from B-DNA are plotted as functions of simulation time in figure $1 \mathrm{a}-\mathrm{c}$ in the presence and absence of CNT. The black line is the DNA transition in the absence of CNT and the blue line is the DNA transition in the presence of CNT. From the variation of the structural parameters, it is clear that the transition happens within 600 ps when CNT is not present. We have also shown instantaneous snapshots of the DNA in the presence of CNT in figure $1 \mathrm{~d}$ at 0,1000 and 2000 ps to highlight the transition from A- to B-DNA. The dsDNA in the presence of $\mathrm{CNT}$ at $0 \mathrm{ps}$ is of A-form and at $1000 \mathrm{ps}$ and $2000 \mathrm{ps}$ it has transformed into B-form. From the plot (figure 1a-c), it is seen that the transition is least affected by the presence of CNT. However, some of the helicoidal parameters such as rise and stretch attain values corresponding to B-DNA little bit slower: it takes $50 \mathrm{ps}$ more in the presence of CNT compared to the case when there is no CNT present. Root mean squared displacement (RMSD) and radius of gyration 
(a)

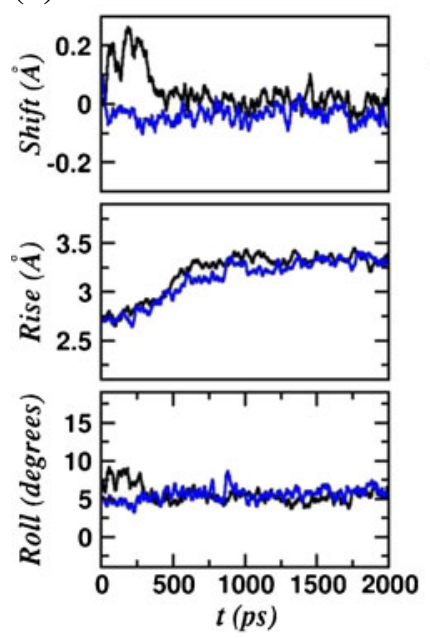

(c)

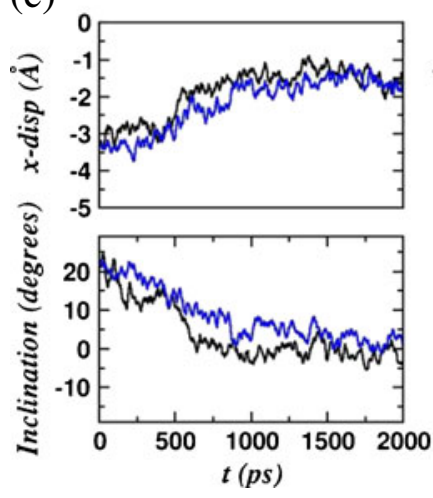

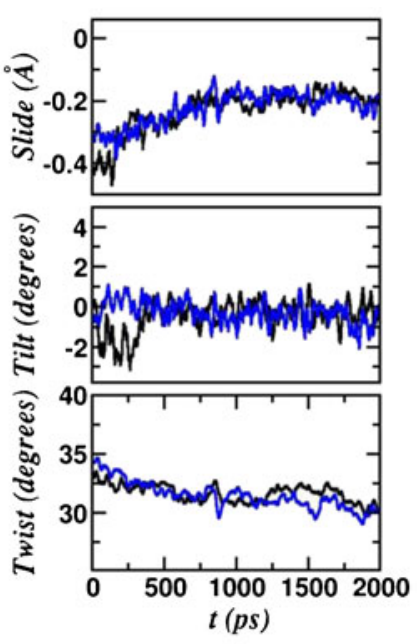

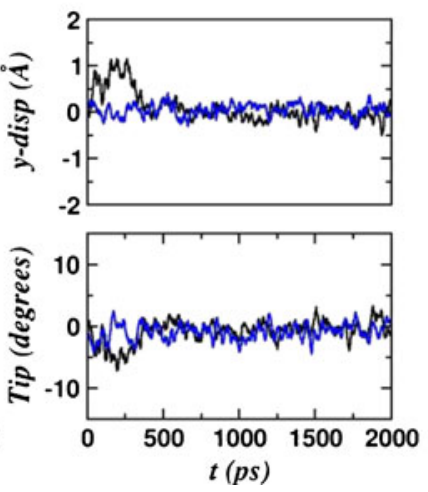

(b)
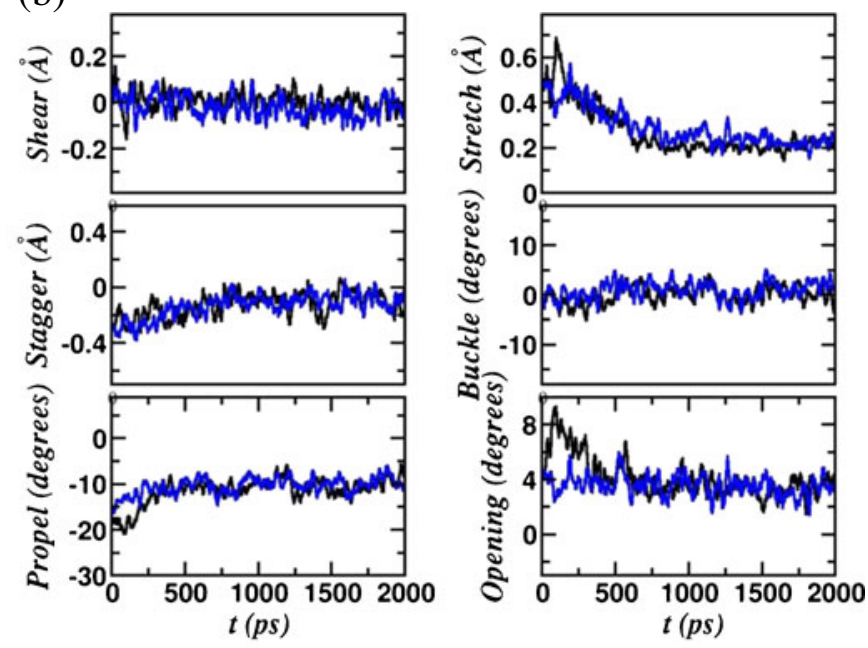

(d)

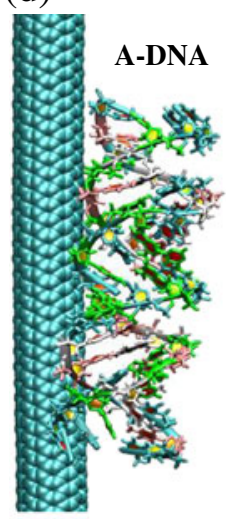

$0 \mathrm{ps}$

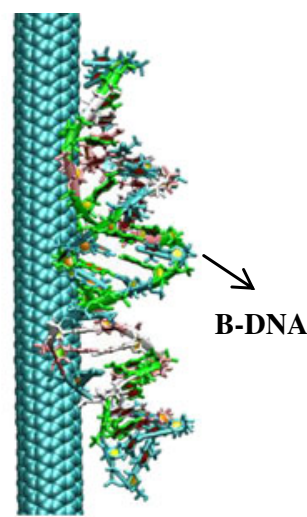

$1000 \mathrm{ps}$

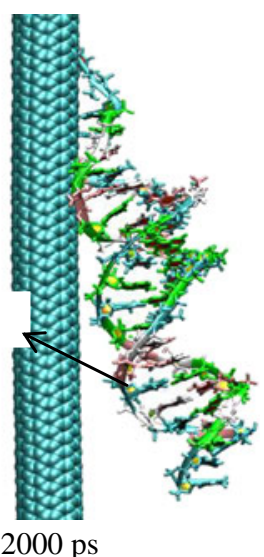

Figure 1. A- to B-DNA transition: (a) inter-base pair, (b) base-base and (c) base pair-axis geometry parameters of A- to B-DNA transition as a function of time. The black line is for DNA transition without CNT and the blue line is in the presence of CNT. We have shown the time scale only for $2000 \mathrm{ps}$, but the B-DNA structure was stable in the simulated time of $10000 \mathrm{ps}$. In the plot of DNA without CNT, as can be seen, all the parameters change and attain a constant value within $600 \mathrm{ps}$, which implies a transition of DNA from A- to B-form in water and neutralizing counterion solution. The presence of CNT has less effect on A- to B-DNA transition, with a little deceleration of the transition.

$\left(\mathrm{R}_{\mathrm{g}}\right)$ of the DNA as a function of time are shown in figure 2a-b. Dramatic increase of RMSD and $\mathrm{R}_{\mathrm{g}}$ within a few hundred picoseconds signifies the occurrence of A-to-B DNA transition both in the presence and absence of CNT.

The mechanism of the A-DNA to B-DNA transition is as follows: in water, the electrostatic screening is large compared to air, which makes water to form hydration shells around $\mathrm{Na}^{+}$ counterions. The formation of hydration shells with $\mathrm{Na}^{+}$ counterions partially inhibits the strong counterion condensation around the negatively charged phosphate atoms. This enhances the electrostatic repulsion between phosphate atoms and increases the phosphate-phosphate distance. Hence, the transition occurs from A-DNA to B-DNA in the presence of water and counterions (Jayaram et al. 1998). The
CNT provides little stability to the initial highly unstable Aform DNA via van der Waals (vdW) interaction with the DNA nucleobases. This vdW interaction, to some extent, competes with the phosphate-phosphate repulsion arising from the backbone. But ultimately the stronger electrostatic repulsion between phosphates wins over the vdW interaction between nucleobases and CNT, resulting in the A-form to make a transition to B-form DNA. However, the transition from A-to-B forms becomes slower in the presence of CNT. In addition to the $\mathrm{vdW}$ interaction, entropy fluctuations in Aform DNA in the initial stage of simulation are suppressed due to the adsorption on the CNT surface, giving stability to A-DNA. We also see that the B-DNA maintains the B-form in the presence of CNT up to $10 \mathrm{~ns}$. However, after the 
(a)

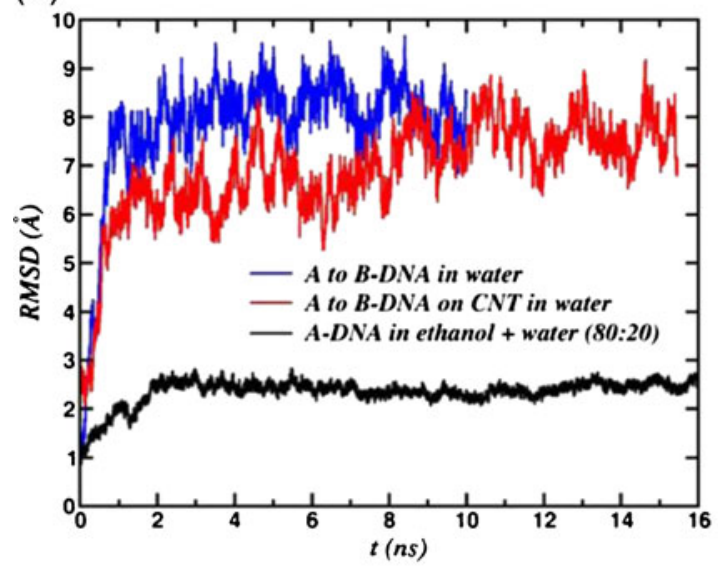

(c)

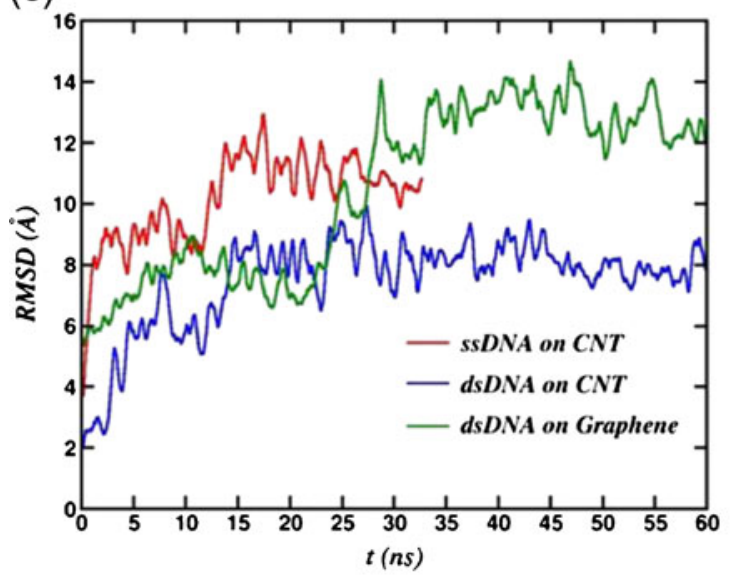

(b)

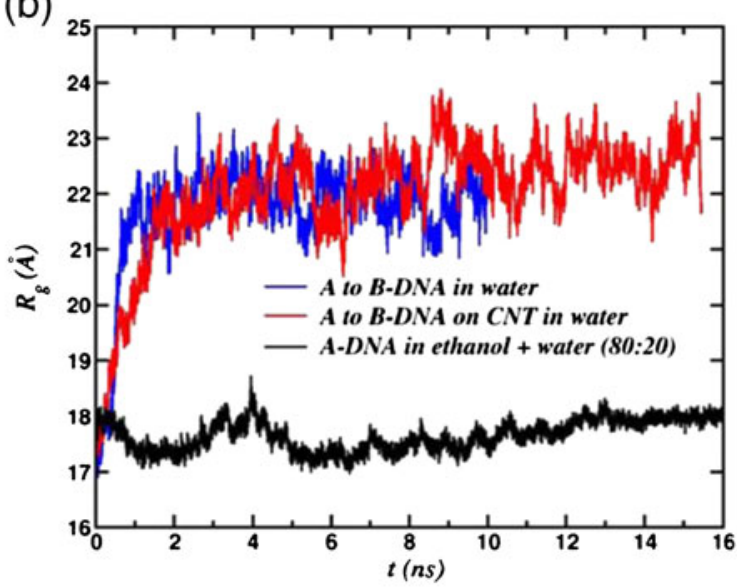

(d)

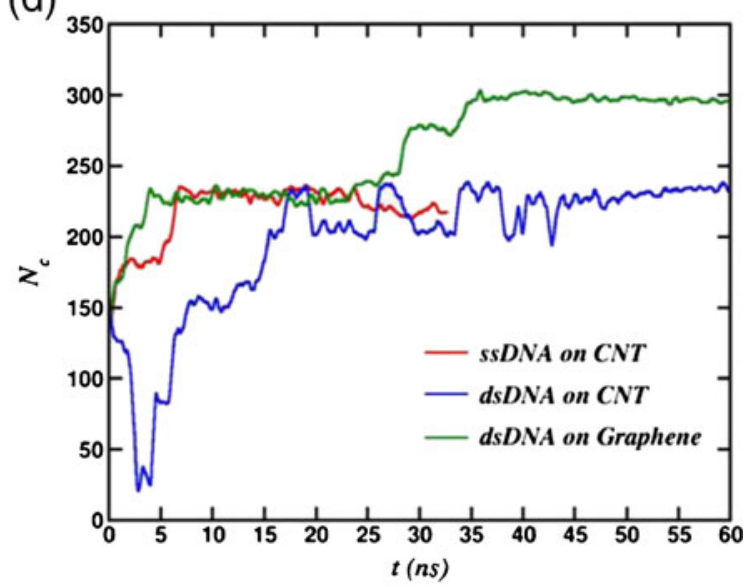

Figure 2. Structural analysis: (a) and (b) are the RMSD and radius of gyration of A- to B-DNA transition in water, in presence of 'CNT+ water' and in the presence of 80:20 ethanol-water solution. As the plots indicate, initial A-DNA transforms into B-DNA in the presence of water and 'CNT+water', whereas A-DNA does not transforms into B-DNA in the presence of ethanol-water solution. (c) and (d) are the RMSD and close contacts of ssDNA on CNT, dsDNA on CNT and dsDNA on graphene. The dsDNA on graphene has maximum RMSD and maximum close contacts compared to ssDNA on CNT and dsDNA on CNT.

transition, CNT can destabilize B-DNA, which is initiated by breaking a few Watson-Crick (WC) H-bonds due to increasing vdW interactions, and eventually in longer time scales, B-DNA can bind to CNT (Santosh et al. 2012).

\subsection{Effect of ethanol on A- to B-DNA transition}

The transition from the stable B-DNA form to other forms such as A-DNA or Z-DNA can be triggered by protein binding or by changing the solvent conditions (Herskovits and Singer 1961; Ivanov et al. 1974; Zimmerman and Pheiffer 1979; Calladine and Drew 1984; Jovin et al. 1987; Dickerson 1992). Ethanol (CH3-CH2-OH) is one of such solvents that can induce the transition from B-form DNA to the A-form DNA. A-DNA is stable in ethanol solvent of proper concentration, whereas B-DNA is stable in aqueous solution at physiological conditions. To see the effect of ethanol on A- to B-DNA transition, we have simulated A-DNA in 80:20 ethanol-water mixtures with neutralizing counterions. The addition of ethanol provides stability to the initial A-DNA, thus inhibiting the transition to B-DNA. Several helicoidal parameters shown in figures $3 \mathrm{a}-\mathrm{c}$ corresponds to A-form of DNA with small fluctuations. Those fluctuations are very small, indicating a very stable A-form in the presence of ethanol solution. The instantaneous snapshots of the DNA shown in figure $3 \mathrm{~d}$ at few nanosecond intervals confirm that the DNA is still in Aform and no transition to B-form has occurred. We have also calculated RMSD and $\mathrm{R}_{\mathrm{g}}$ of A-DNA in 80:20 ethanol-water mixture as shown in figure $2 \mathrm{a}-\mathrm{b}$ when no A-to-B transition has occurred. These data support the stability of A-DNA in 
(a)

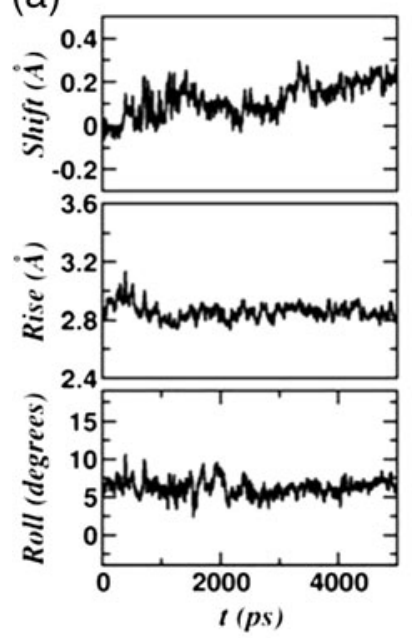

(c)

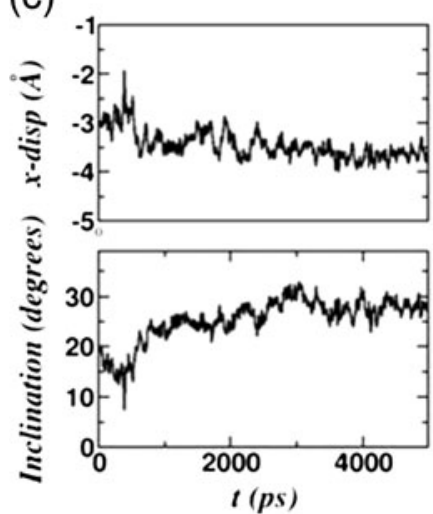

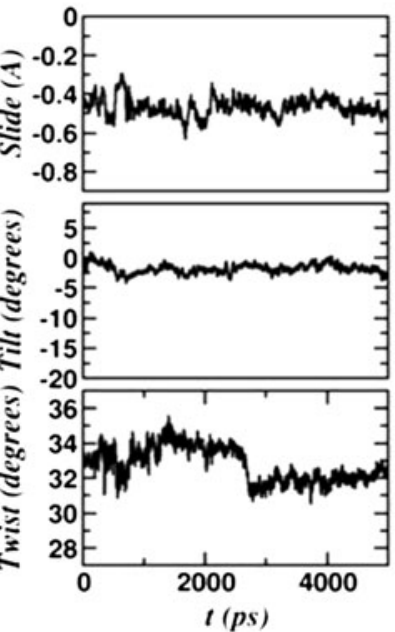

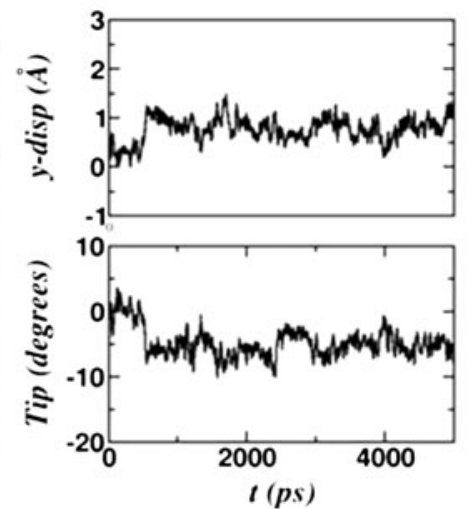

(b)

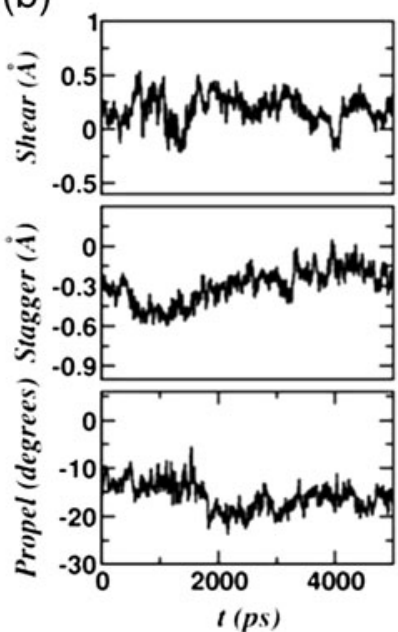

(d)

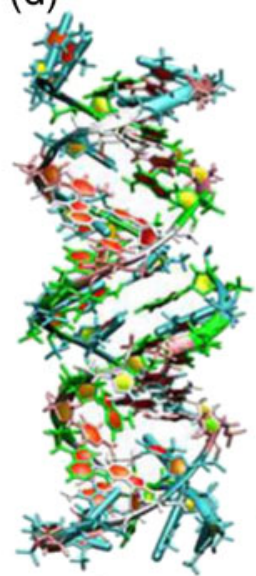

0 ns

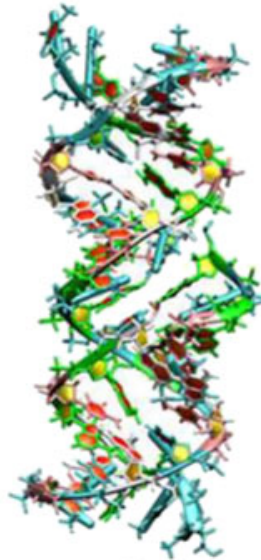

$5 \mathrm{~ns}$

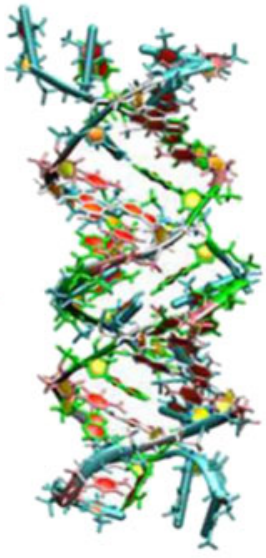

$10 \mathrm{~ns}$

Figure 3. Stable A-DNA in 80:20 ratios of ethanol and water as a solution: (a) inter-base pair, (b) base-base and (c) base pair-axis helicoidal parameters as a function of time show that A-DNA is stable with all the parameters corresponding to A-DNA from in ethanol solution. (d) Snapshots shown at 0, 5 and $10 \mathrm{~ns}$ indicate that the A-DNA remains stable in A-form and does not transform into B-form as was the case in the absence of ethanol.

ethanol-water mixture solution. In the presence of water, the formation of hydration shells around $\mathrm{Na}^{+}$counterions partially inhibits the strong counterion condensation around the negatively charged phosphate atoms that increases electrostatic repulsion between phosphate atoms. This increases the phosphate-phosphate distance, and hence favours a stable B-DNA in the presence of water and counterions. The dielectric constant of ethanol is 24 and that of water is 80 . Hence, the dielectric constant of the mixture solution consisting of ethanol and water is less than that of water (Reynolds and Hough 1957). Hence, when ethanol is added to the solution of A-DNA, water and counterions, the electrostatic potential affects the hydration shells, and hence $\mathrm{Na}^{+}$counterions have greater improvement in counterion condensation with phosphate groups, resulting in decrease of phosphate-phosphate repulsion. So the ethanol solution favours A-DNA form over B-DNA in ethanolwater solution. The interaction of A-DNA with CNT in the presence of ethanol-water mixture is under study.

\section{DNA interaction with CNT and graphene}

\subsection{Adsorption of ssDNA and dsDNA on CNT}

Nucleic acids such as ssDNA and dsDNA adsorption on CNT have been studied by several groups that have found promising applications in CNT separation and sensors within cells (Zheng et al. 2003a; Zheng et al. 2003b; Chou et al. 2004; Heller et al. 2006; Zhao and Johnson 2007; Johnson 
et al. 2008). In contrast, not much attention has been paid to study the interaction of siRNA with CNTs. Only recently have we studied siRNA interaction with CNTs of various diameters (Santosh et al. 2012). We have studied the adsorption of ssDNA and dsDNA with sticky-ends on $(6,6)$ CNT at $300 \mathrm{~K}$. The sequence of dsDNA with sticky-ends is same as siRNA except for the presence of thymine in place of uracil. The dsDNA sequence used in our simulation is d(TT AGA CAG CAT ATA TGC TGT CT) ${ }_{2}$ and that of ssDNA is d(TT AGA CAG CAT ATA TGC TGT CT), which is the same as one of the strands of the dsDNA. Instantaneous snapshots of ssDNA and dsDNA adsorption on CNT are shown in figure $4 \mathrm{a}$ and $\mathrm{b}$. As can be seen from the figure $4 \mathrm{a}$, ssDNA adsorbs strongly and wraps around the CNT surface with almost all nucleobases of ssDNA interacting with CNT via $\mathrm{vdW}$ dispersive forces. The snapshot shown here was one of the many binding modes of ssDNA on CNTs. To characterize the binding affinity as well as the conformational changes of ssDNA and dsDNA while binding with CNTs, we calculated the RMSD and number of close contacts (number of DNA atoms within $5 \AA$ cut-off from CNT atoms), which are shown in figure $2 \mathrm{c}-\mathrm{d}$. As shown in figure $2 \mathrm{c}-\mathrm{d}$, the RMSD and close contacts attain a constant value, indicating a stable bound configuration of ssDNA on the CNT. For ssDNA, the average RMSD is $11 \AA$ and the number of close contacts is 230 within $5 \AA$ from the CNT surface. This indicates a strong wrapping of ssDNA around the CNT. However, the dsDNA gets adsorbed to CNT with partial unzipping in the long simulation time of $70 \mathrm{~ns}$. In contrast, siRNA binds to CNT through the unzipping of two strands. This is to some extent due to the relatively strong A-T base pair interaction energy compared to the A-U base pair interaction energy (Sponer et al. 2004; Santosh et al. 2012). A similar kind of dsDNA adsorption on CNT was reported in an earlier simulation study (Zhao and Johnson 2007). However, there is more fluctuation in the RMSD due to lower binding to CNT. The RMSD of dsDNA when adsorbed to CNT is only $8 \AA$, which is less compared to the RMSD of $11 \AA$ for ssDNA on CNT. The structural deformation of dsDNA is very less compared to ssDNA. Our simulation studies give microscopic picture of the ssDNA-CNT and dsDNA-CNT hybrid.

\subsection{Translocation of ssDNA and dsDNA inside CNT}

Earlier Gao et al. (2003) reported the spontaneous translocation of 8 bases long ssDNA through $(10,10)$ a CNT of diameter $1.36 \mathrm{~nm}$. In their study, they used GROMOS FF (Vangunsteren and Berendsen 1987). However, to the best of our knowledge there exist no simulation studies which demonstrate the translocation of ssDNA or dsDNA through CNT using more accurate force fields like AMBER or CHARMM (Case et al. 2006; Brooks et al. 2009). In the process of complete understanding of DNA-CNT interactions, we also studied the translocation of ssDNA and dsDNA in a $(20,20)$ CNT using AMBER FF03 (Duan

(a)

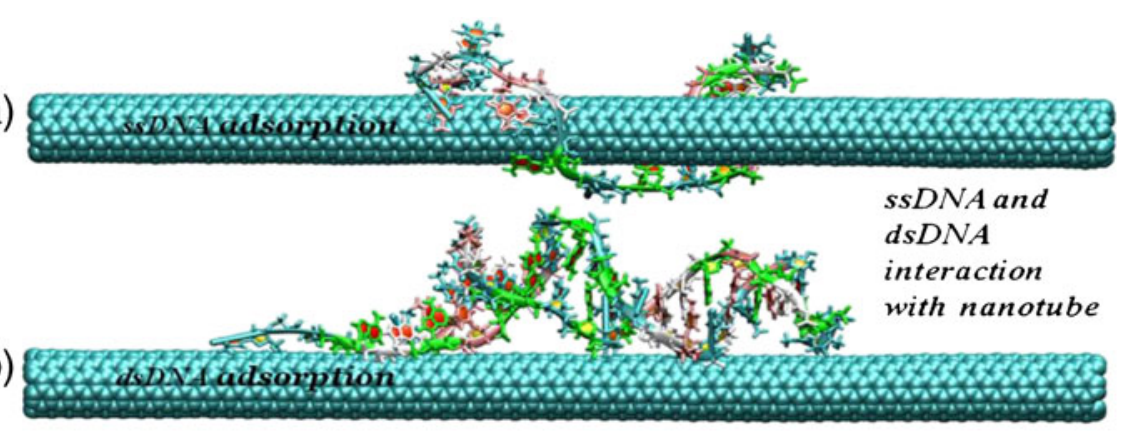

(c)

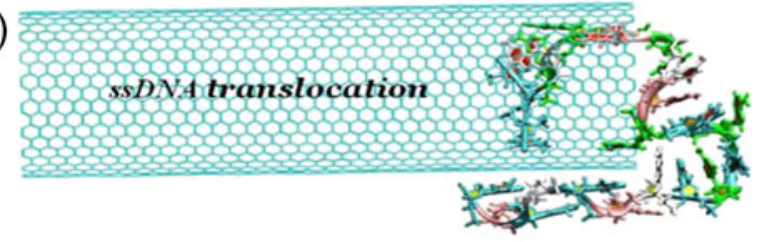

(d)

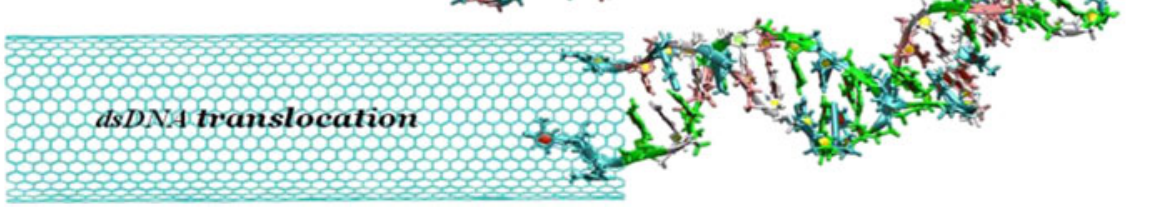

Figure 4. Adsorption and translocation of ssDNA and dsDNA with carbon nanotube. ssDNA strongly adsorbs and also translocates into carbon nanotube, whereas dsDNA only adsorbs but not translocate into $(20,20)$ CNT. 
et al. 2003). The $(20,20)$ CNT has a diameter of $2.7 \mathrm{~nm}$. Note that in our simulation, we used 22 bases long ssDNA in contrast to the 8 bases long ssDNA used in the study of Gao et al. (2003). Figure 4c shows the results on translocation of ssDNA into $(20,20)$ CNT. Nearly half of the 22 nucleobases of the ssDNA enter inside the hydrophobic cavity of $(20,20)$ CNT within $20 \mathrm{~ns}$ and remain stable there in that conformation for a long time without translocating. The dispersion interaction of nucleobases with the aromatic rings of CNT makes the ssDNA get 'stuck' in a metastable local minimum of free energy surface and prevents complete translocation after half of the nucleobases enter the CNT. The other half of the ssDNA not inside the CNT wraps around the surface of the CNT. This structure is stable after 20 ns with minimal deviations in structural parameters such as RMSD and close contacts. For comparison, we have also simulated the translocation of dsDNA of 22 base pairs long inside $(20,20)$ CNT. Figure 4d shows the instantaneous snapshot of the system after 30 ns. However, dsDNA does not enter the CNT of the same diameter where part of ssDNA translocation was observed. A molecular level understanding of these phenomena is not clear yet. This could be due to the large free energy barrier for the entry of dsDNA into the interior of CNT. As shown in figure $4 d$, only one to two nucleobases that are near to the CNT unzip by breaking WC H-bonds between them and interact with CNT. The unzipping of few base pairs and subsequent binding to the CNT surface may be preventing the dsDNA from entering the nanotube, and so no further translocation is observed. However, it would be interesting to study the translocation of dsDNA through CNT by introducing electric fields or pressure gradients or flow through nanotubes.

\subsection{Adsorption of dsDNA on graphene}

Graphene is a one-atom-thick 2D material of carbon with extraordinary properties due to which it can be used for many applications (Novoselov et al. 2004; Zhang et al. 2005; Stankovich et al. 2006; Geim and Novoselov 2007; Castro Neto et al. 2009). DNA sequencing is one of the promising areas where graphene can effectively be used, as has been demonstrated by several recent works (Garaj et al. 2010; Schneider et al. 2010; Merchant et al. 2010). The microscopic understanding of DNA interaction with graphene is essential for such future applications. We have studied the interaction of dsDNA with graphene to understand the adsorption phenomena. In figure 5, we show instantaneous snapshots of dsDNA on graphene in few nanosecond intervals as the binding progresses. The sequence of dsDNA used in our simulation has two stickyends which take part in $\pi-\pi$ stacking with graphene. During the course of simulation, a few base pairs at two ends get unzipped and these unzipped base pairs facilitate better binding with the graphene. The binding affinity of dsDNA is more with graphene compared to its binding with CNT.

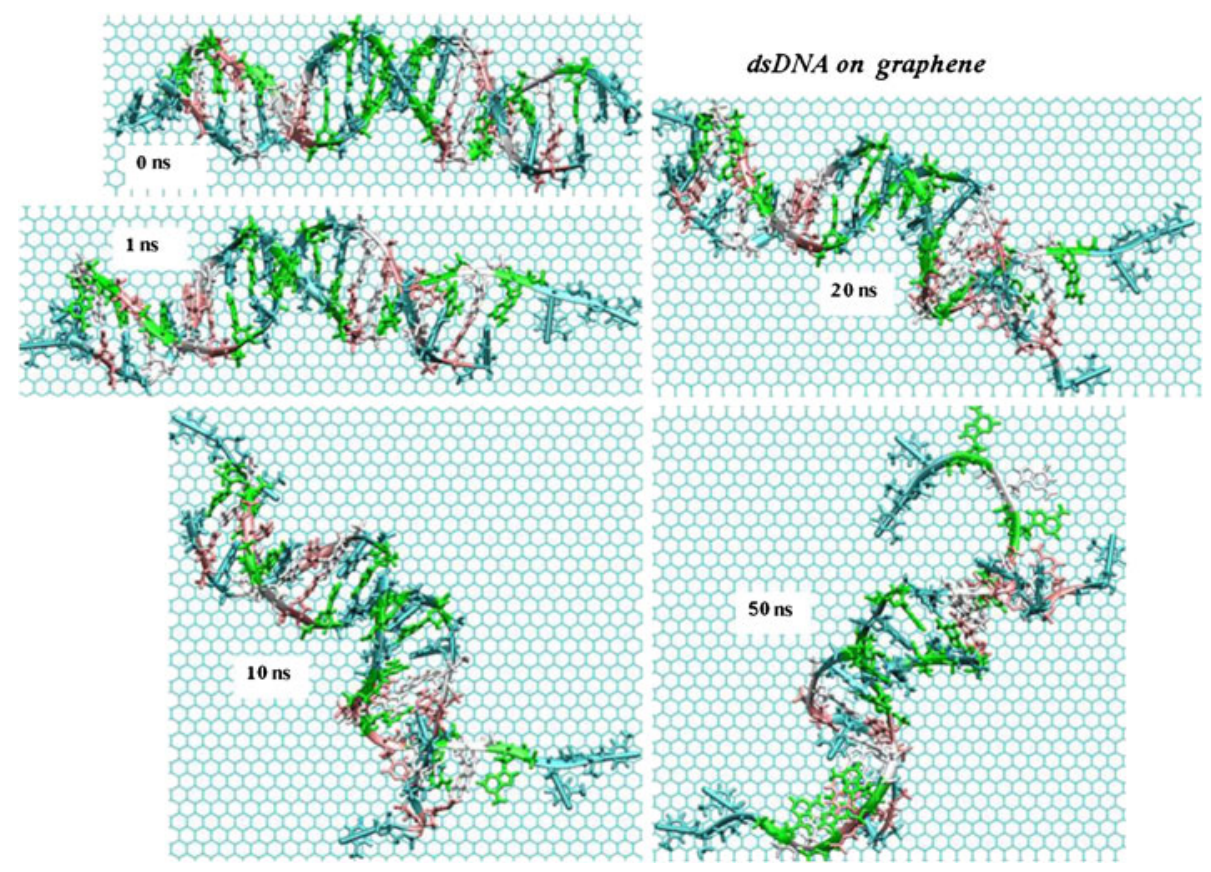

Figure 5. Instantaneous snapshots of dsDNA on graphene. A few base pairs at the two ends get unzipped and interact with graphene via van der Waals to bind to graphene. 
Within $6 \mathrm{~ns}$, approximately 8 of total 48 WC H-bonds in dsDNA get unzipped and remain constant at $40 \mathrm{H}$-bonds throughout the simulation time of $80 \mathrm{~ns}$. We have also calculated some of the structural parameters such as the number of close contacts and RMSD of dsDNA to probe the stability of dsDNA-graphene hybrid and have shown them in figure $2 \mathrm{c}-\mathrm{d}$. These calculations reveal that the hybrid is very stable after initial unzipping and adsorption of few base pairs of dsDNA. In the stable configuration, the average number of close contacts of dsDNA is 240 within a cut-off of $5 \AA$ from graphene surface and the average RMSD of dsDNA is $8.5 \pm 0.6 \AA$. As the binding of dsDNA is more on graphene, the dsDNA structure deforms largely with respect to its initial crystal structure, leading to a large value of RMSD. The WC H-bonds and structural parameters such as RMSD quantify the binding of dsDNA on graphene. The binding property of dsDNA on graphene can be used in graphenebased sensing and DNA sequencing applications (Garaj et al. 2010; Merchant et al. 2010; Schneider et al. 2010).

\section{Nucleic acids interaction with dendrimers}

\subsection{Interaction of DNA with dendrimers}

4.1.1 DNA compaction: Dendrimers are a class of organic molecule with a well-defined structures and molecular weights. This mono-dispersed molecule grows in a stepwise manner (Frechet 1994). As a function of generation, a dendrimer has exponentially growing numbers of primary and tertiary amines. Depending on the $\mathrm{pH}$ of the solvent, the primary and tertiary amines of the dendrimer can be protonated or deprotonated. At physiological $\mathrm{pH}$, for example, the PAMAM dendrimer is positively charged and can effectively bind negatively charged DNA, and so can be used as gene delivery material inside mammalian cells (Zimmerman et al. 1996; Bosman et al. 1999; Grayson and Frechet 2001). Lower levels of toxicity (KukowskaLatallo et al. 1996) and high positive surface charge of protonated PAMAM dendrimers make them suitable candidates for gene transfection (Haensler and Szoka 1993; Dufes et al. 2005). A few computational studies using all atom molecular dynamics (MD), coarse-grained MD and Brownian dynamics simulations (Lyulin et al. 2008; Larin et al. 2009b; Pavan et al. 2010) already exist as background references.

4.1.2 Structural aspect: In the recent past a number of theoretical and computational studies on structural properties of polyelectrolyte-dendrimer complexation under different conditions have been reported (KukowskaLatallo et al. 1996; Harries et al. 1998; Scherrenberg et al. 1998). Using Monte Carlo (MC) simulation, Welch and Muthukumar (Welch and Muthukumar 2000) first showed the complexation between a charged linear chain and a model poly-propyle-imine (PPI) dendrimer with charged terminal groups under various salt concentrations. In their study, it was predicted that DNA adsorption is dependent on the $\mathrm{pH}$ of the solvent, size of the dendrimer, length of the DNA and the charge ratio of the DNA and dendrimer. In the same year, Tomalia et al also reported various mechanisms of DNA complexation with low and high generations of PAMAM dendrimers using spectroscopic observation (Ottaviani et al. 2000). Later, Maiti and Bagchi reported the first atomistic simulations of DNA compaction by dendrimers (Maiti and Bagchi 2006). An extensive study of the complexation of a 38 base pairs ssDNA with PAMAM dendrimers of generations $\mathrm{G} 2-\mathrm{G} 4$ at physiological $\mathrm{pH}$ was reported. It was shown that ssDNA coils around the PAMAM dendrimer of generation 4 where the electrostatic interaction between the positive overcharges on the dendrimer and negative charges in the DNA backbone help to compensate the penalty due to the bending of the DNA. Figure 6a shows the instantaneous snapshot of the ssDNAdendrimer complex for G4 PAMAM dendrimer at intervals of few nanoseconds. In the case of G3 and G2, partial adsorption of the ssDNA on the dendrimer was observed as seen in figures $6 \mathrm{~b}$ and $\mathrm{c}$. Subsequently, we have extended our effort to study the interaction of dsDNA with PAMAM dendrimer of generations G3-G5 (Nandy and Maiti 2011). Various binding modes of complexation between PAMAM (generations G3-G5) and the 38 base pairs dsDNA at neutral $\mathrm{pH}$ was reported and have been shown in figures $7 \mathrm{a}-\mathrm{c}$. Semiflexible negatively charged dsDNA have a Kuhn segment length of $106 \mathrm{~nm}$ and a linear charge density equal to electronic charge per $1.7 \AA$. It has been reported that dsDNA having length shorter than its persistence length $50 \mathrm{~nm}$ $(\sim 150 \mathrm{bp})$ is likely to behave as a rigid rod (Bielinska et al. 1997). Nandy and Maiti (Nandy and Maiti 2011) showed that a 38 base pairs dsDNA with a length of $12 \mathrm{~nm}$ can wrap completely on the surface of G5 PAMAM dendrimer (figure 7a). Given the fact that a 38-base-pair-long dsDNA having a length of $12 \mathrm{~nm}$, to good approximation, can be considered to be rigid rod, this is a very surprising result. The binding energy analysis revealed that the binding is driven by electrostatic energy (Fant et al. 2008) and the larger the positive charges on the dendrimer, stronger is the binding interaction. Our finding shows that DNA is not able to wrap the smaller generation dendrimers like G2 and G4, which is in agreement with the experimental results of Tomalia and co-workers (Ottaviani et al. 2000). Lowergeneration dendrimers like G3 and G4 are able to wrap the DNA only partially as depicted in figure $7 \mathrm{~b}-\mathrm{c}$. It is known that the charge ratio $(\mathrm{P} / \mathrm{N})$ between positively charged dendrimer and negatively charged DNA is critical for the shape and structure of the complex. When $\mathrm{P} / \mathrm{N}$ is less than 1 , as in the case of lower-generation dendrimers (G3 and G4) with only few cationic groups, DNA interaction with dendrimer is 


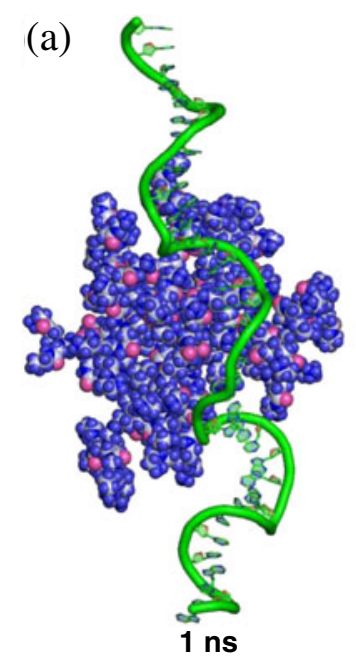

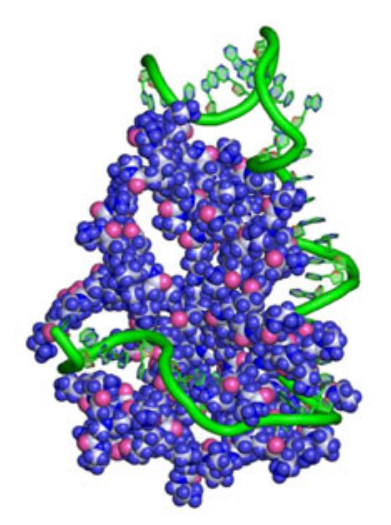

13 ns

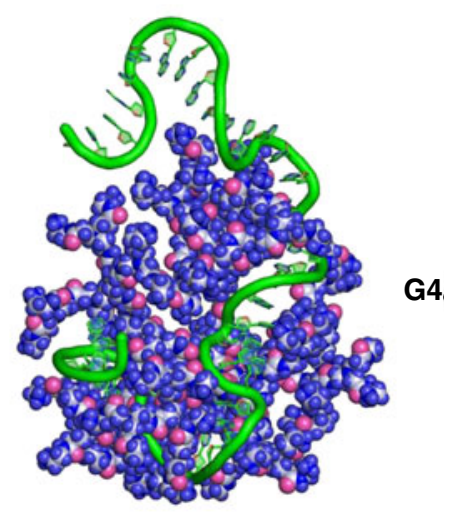

$18 \mathrm{~ns}$

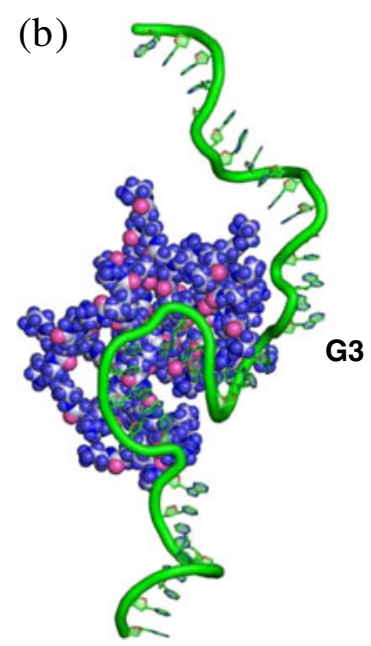

(c)

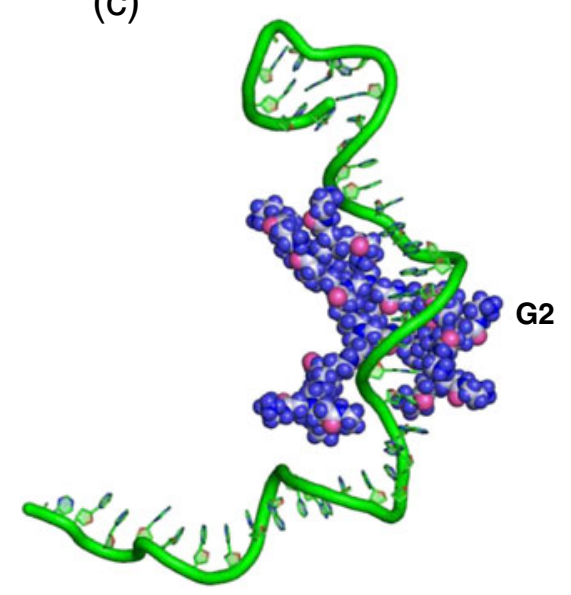

Figure 6. (a) Various binding modes of ssDNA-G4 dendrimer complexation at the interval of a few nanoseconds. (b) Structure of ssDNA binding with G3 and (c) structure of ssDNA binding with G2.

very similar to interaction of spermine or hexamine cobalt (Zinchenko and Chen 2006). In contrast, DNA wraps around the higher-generation dendrimer like G5 and resembles to the DNA wrapping around nucleosomes.

Regarding the dendrimer's conformational changes, a few important observations have been made in this study. During the complex formation, the dendrimer expands noticeably in the early stage to make large number of contacts with DNA. In the next several nanoseconds, the dendrimer tries to optimize its binding affinity by finding a suitable binding site on the DNA surface. During this process of binding, the lowergeneration dendrimer faces a significant amount of structural deformation compared to the higher-generation dendrimer. We have calculated the asphericity $(\delta)$ parameter to determine the structural deformation of dendrimer (Maiti et al. 2004). Asphericity results reflect that the G3 dendrimer undergoes considerable amount of distortion from the spherical shape compared to the G4 and G5 dendrimer (Nandy and Maiti 2011). Radius of gyration $\left(R_{g}\right)$ as a function of time gives the structural evolution of the DNAdendrimer complex. This is also a measure of the conformational change of DNA-dendrimer during the complexation process. Figure $8 \mathrm{a}$ and $\mathrm{c}$ gives the time evolution of $\mathrm{R}_{\mathrm{g}}$ for dsDNA-G3 dendrimer and dsDNA-G5 dendrimer at neutral $\mathrm{pH}$. Density distribution is another important structural parameter which reveals the internal structural arrangement of DNA and dendrimer inside the complex as well as distribution of water surrounding the complex. Corresponding density distribution profiles $\rho(\mathrm{r})$ are shown in figure $8 b$ and $d$. Note that for the G5-dsDNA complex, we have a compact complex and the size of the complex is very similar to the size of the dendrimer. However, this leads to more penetration of DNA inside the dendrimer, which can make the subsequent DNA release difficult. One should keep in mind that an efficient gene delivery carrier should release 
(a)

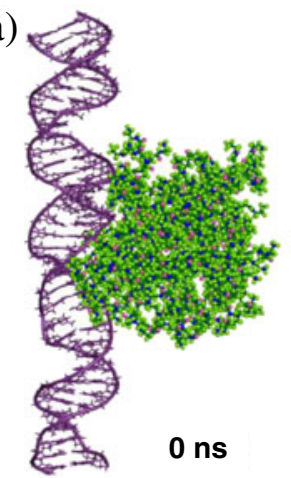

(b)

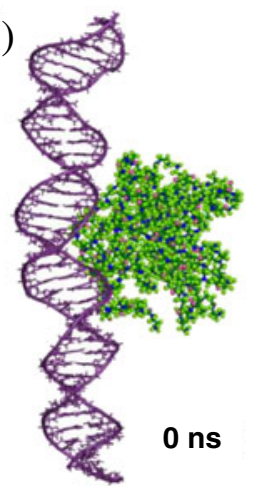

(c)

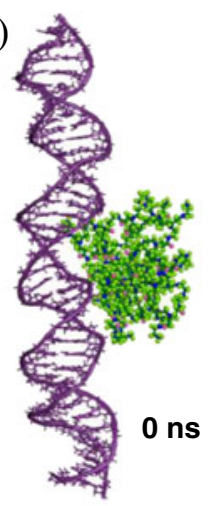

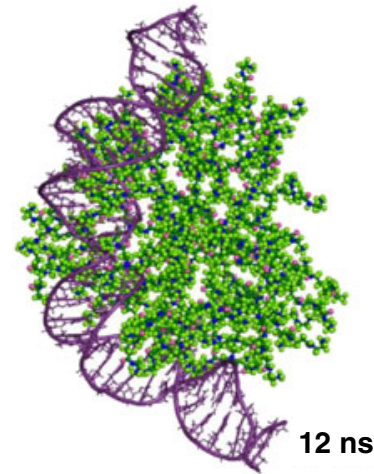
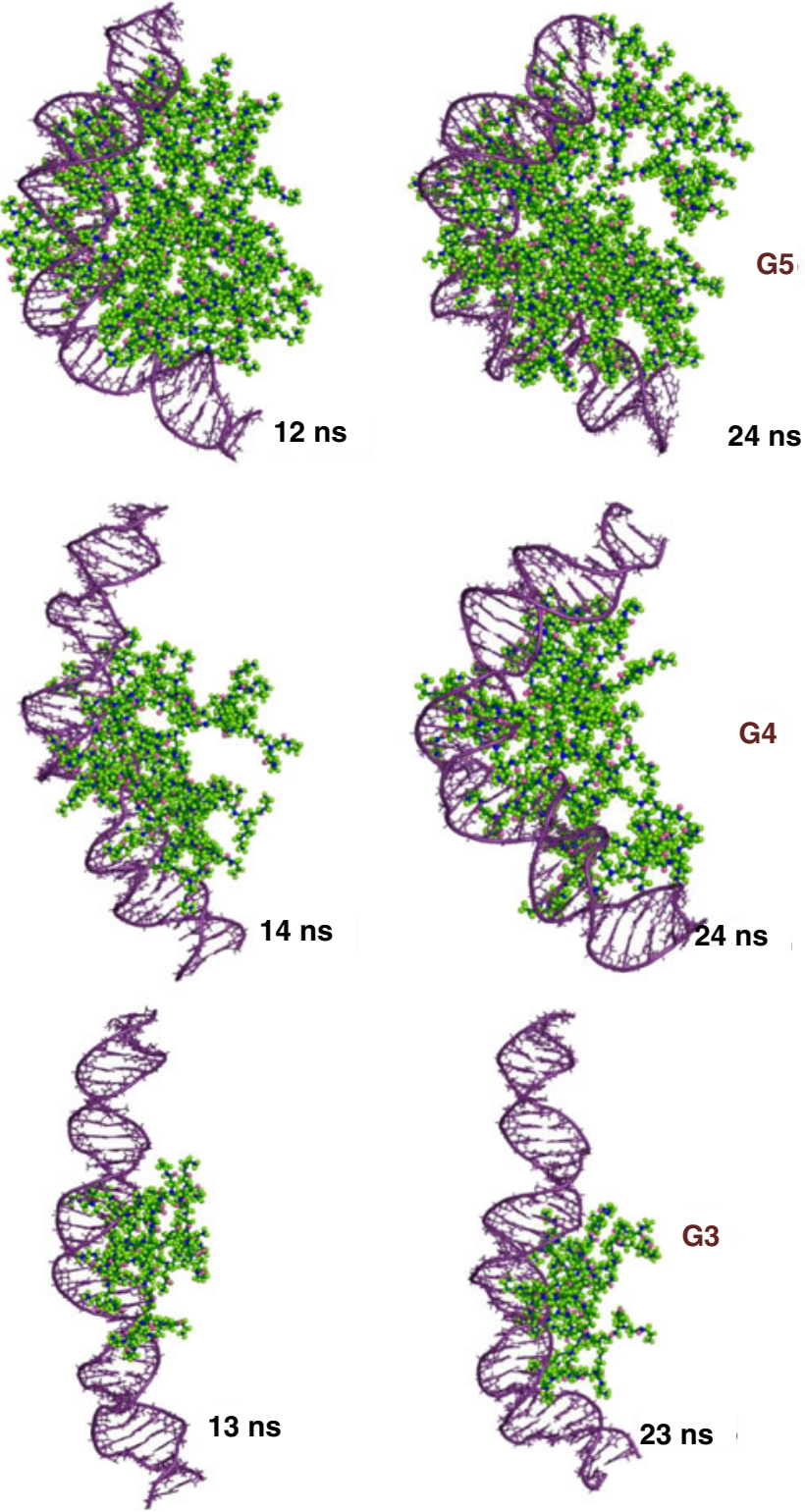

G5

G4

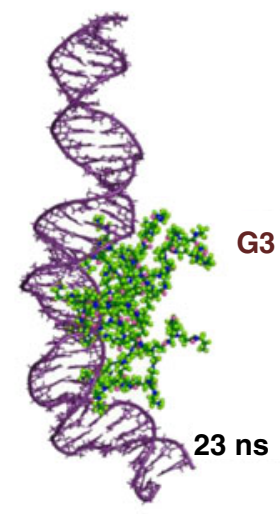

Figure 7. (a-c) Structure of DNA-dendrimer complex during various stages of complex formation at the interval of few nanoseconds for G5, G4 and G3 PAMAM respectively.

the DNA easily from the complex prior to nuclear entry. Excess DNA penetration can create complicacy at the time of DNA release.

4.1.3 pH dependence: The PAMAM dendrimer is uncharged at high $\mathrm{pH}(\sim 10)$, and as a result the complex formation with ssDNA does not occur at that condition as can be seen from figure 9a. Instead, the DNA moves away from the dendrimer as time progresses. At neutral $\mathrm{pH}(\sim 7)$, dendrimer terminal amines become protonated and strong electrostatic attraction between the positively charged dendrimer and negatively charged ssDNA helps to wrap the DNA around the dendrimer. Figure $9 \mathrm{~b}$ shows the wrapping of ssDNA on the surface of protonated $\mathrm{G} 4$ dendrimer at neutral $\mathrm{pH}$. At low $\mathrm{pH}$ $(\sim 3)$ both the primary and tertiary amines get protonated, thus making the dendrimer even more positively charged. Figure $9 \mathrm{c}$ gives a representative snapshot of the complex at low $\mathrm{pH}$. At low $\mathrm{pH}$, the penetration of DNA inside the dendrimer is less than the case of neutral $\mathrm{pH}$. At high salt concentration (low $\mathrm{pH}$ ), negative $\mathrm{Cl}^{-}$ions of the solvent accumulate on the surface as well as the interior of the dendrimer, which in turn make the dendrimer neutral and weaken the binding between the DNA and dendrimer. As mentioned earlier, excessive penetration of DNA inside the 
(a)

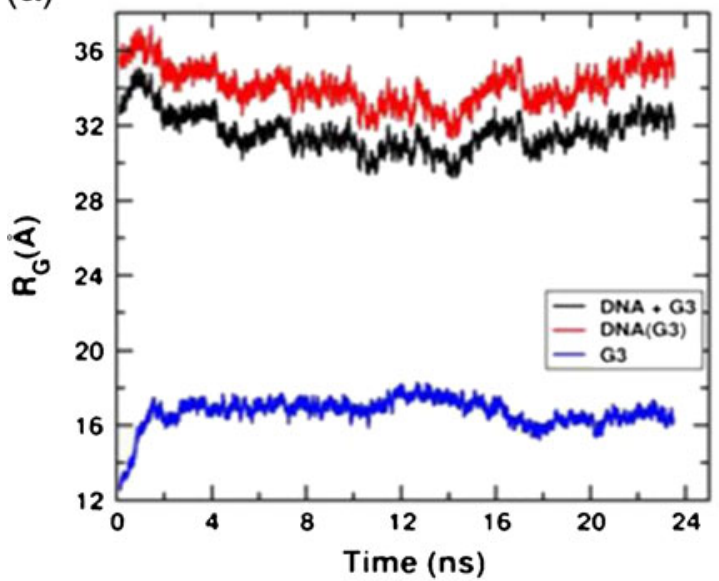

(c)

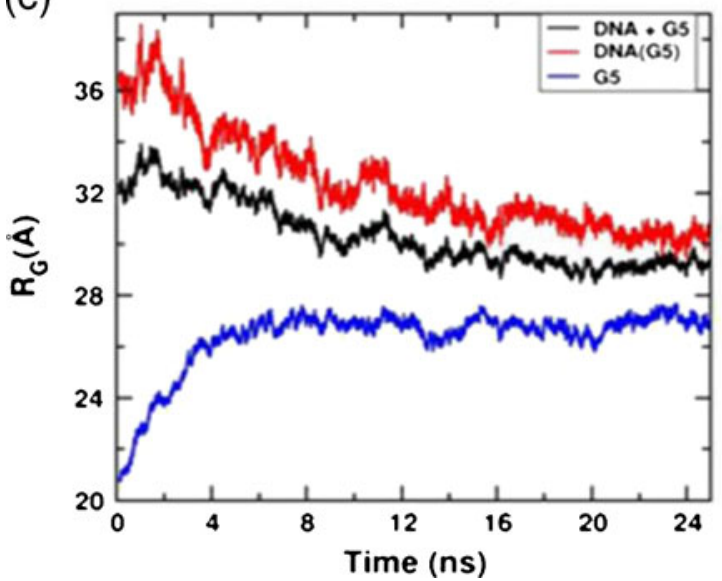

(b)

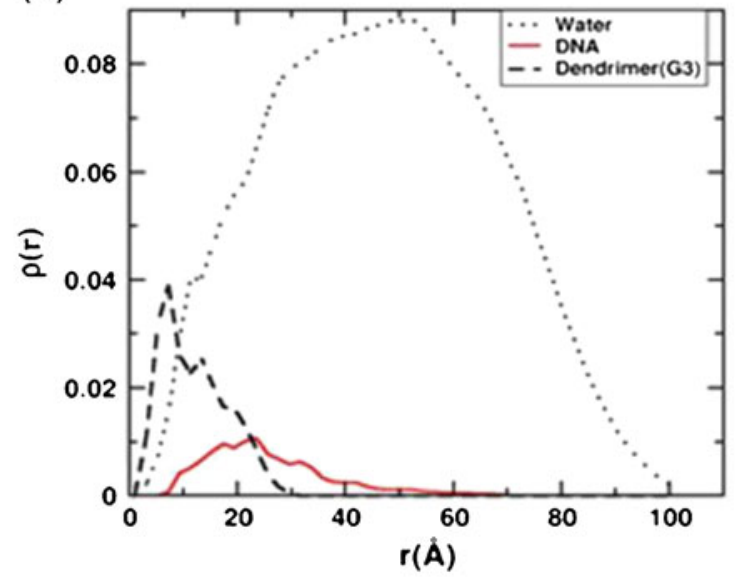

(d)

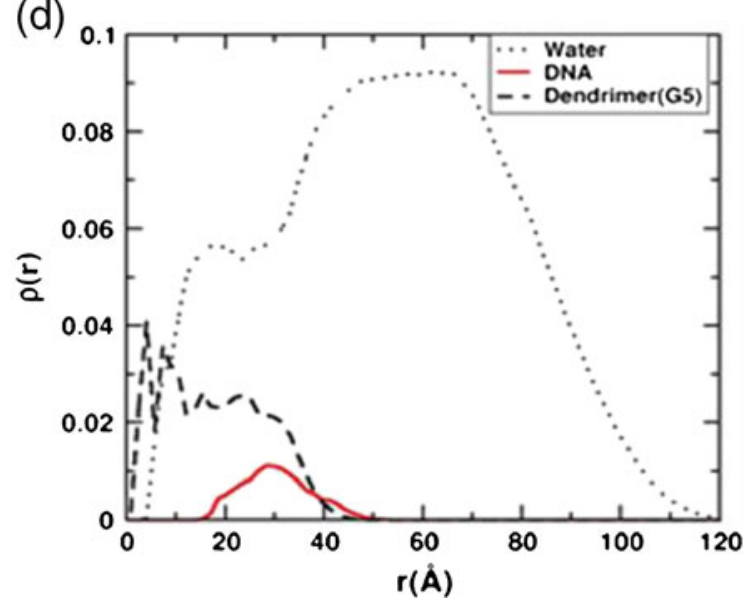

Figure 8. (a) and (c): Time evolution of radius of gyration $\left(\mathrm{R}_{\mathrm{g}}\right)$ of DNA, dendrimer and the complex during the complexation with $\mathrm{G} 3$ and G5 dendrimer respectively at neutral pH. (b-d) Density distribution for DNA, dendrime and water corresponding to G3 and G5 generations respectively and the distribution has been calculated with respect to centre of mass of dendrimer. Figure taken from Nandy and Maiti (2011) with permission from the ACS.

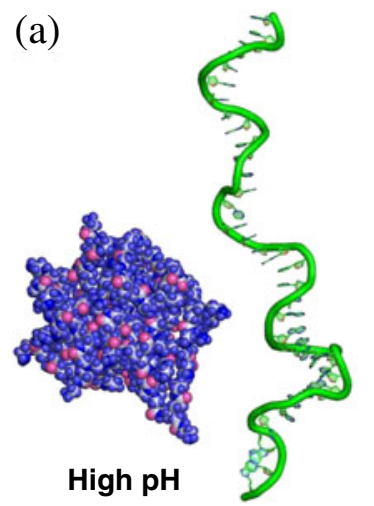

(b)

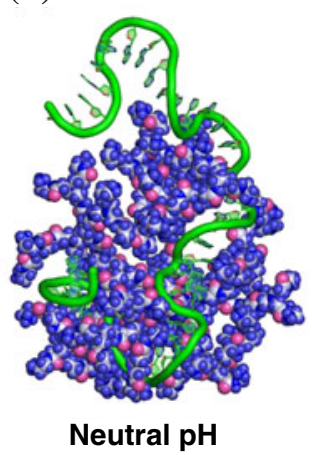

(c)

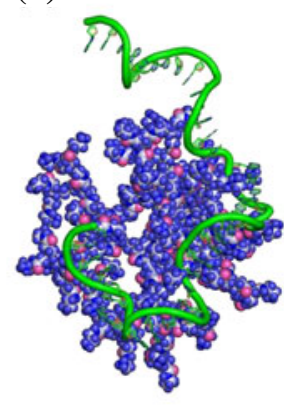

Low pH

Figure 9. Equilibrium snapshot of ssDNA-dendrimer complexes at (a) high, (b) neutral and (c) low pH. The dendrimer has been shown in sphere representation in different shades of blue and pink, while the DNA is shown in the cartoon representation. 
Table 1. Binding free energy using MM-PB/GB SA and 2PT methods for dsDNA-dendrimer complex

\begin{tabular}{lcccc}
\hline Complex & Electrostatic energy $(\mathrm{kcal} / \mathrm{mol})$ & Energy $(\mathrm{kcal} / \mathrm{mol})$ & $-\mathrm{T} \Delta \mathrm{S}(\mathrm{kcal} / \mathrm{mol})$ & $\Delta \mathrm{G}(\mathrm{kcal} / \mathrm{mol})$ \\
\hline G3+DNA & $-1639.76 \pm 703.61$ & $-253.78 \pm 5.75$ & 7.506 & -246.27 \\
G4+DNA & $-6019.65 \pm 559.28$ & $-789.71 \pm 10.78$ & 53.191 & -736.52 \\
G5+DNA & $7429.57 \pm 618.87$ & $-1283.42 \pm 16.07$ & 52.862 & -1230.56 \\
G5+DNA $(50 \mathrm{mM})$ & $-4220.18 \pm 327.73$ & $-1175.48 \pm 14.57$ & 10.782 & -1164.69 \\
\hline
\end{tabular}

dendrimer can create difficulty at a later stage of DNA release in case of gene therapy. So lower penetration of DNA inside dendrimer at low $\mathrm{pH}$ can mediate high DNA delivery activity inside the cell.

4.1.4 DNA bending: When a negatively charged DNA concentrates near the surface of a positively charged dendrimer, the size and the geometry of that dendrimer plays a crucial role in DNA wrapping. During the wrapping process, DNA bends significantly and this bending of DNA depends on various parameters like dendrimer-DNA charge ratio, dendrimer generation, etc. We calculated various helicoidal parameters to characterize the conformational change of DNA as well as the bending of DNA helix. When a helical axis produces negative roll angle, that indicates the bending is towards the DNA minor groove, whereas bending toward a major groove produces a positive roll angle. By calculating different bending parameters (global bending, DNA strand shortening, local bending) Nandy and Maiti (Nandy and Maiti 2011) showed that DNA bends significantly more when complexed with a higher-generation dendrimer like G5 compared to lower-generation dendrimer such as G3.
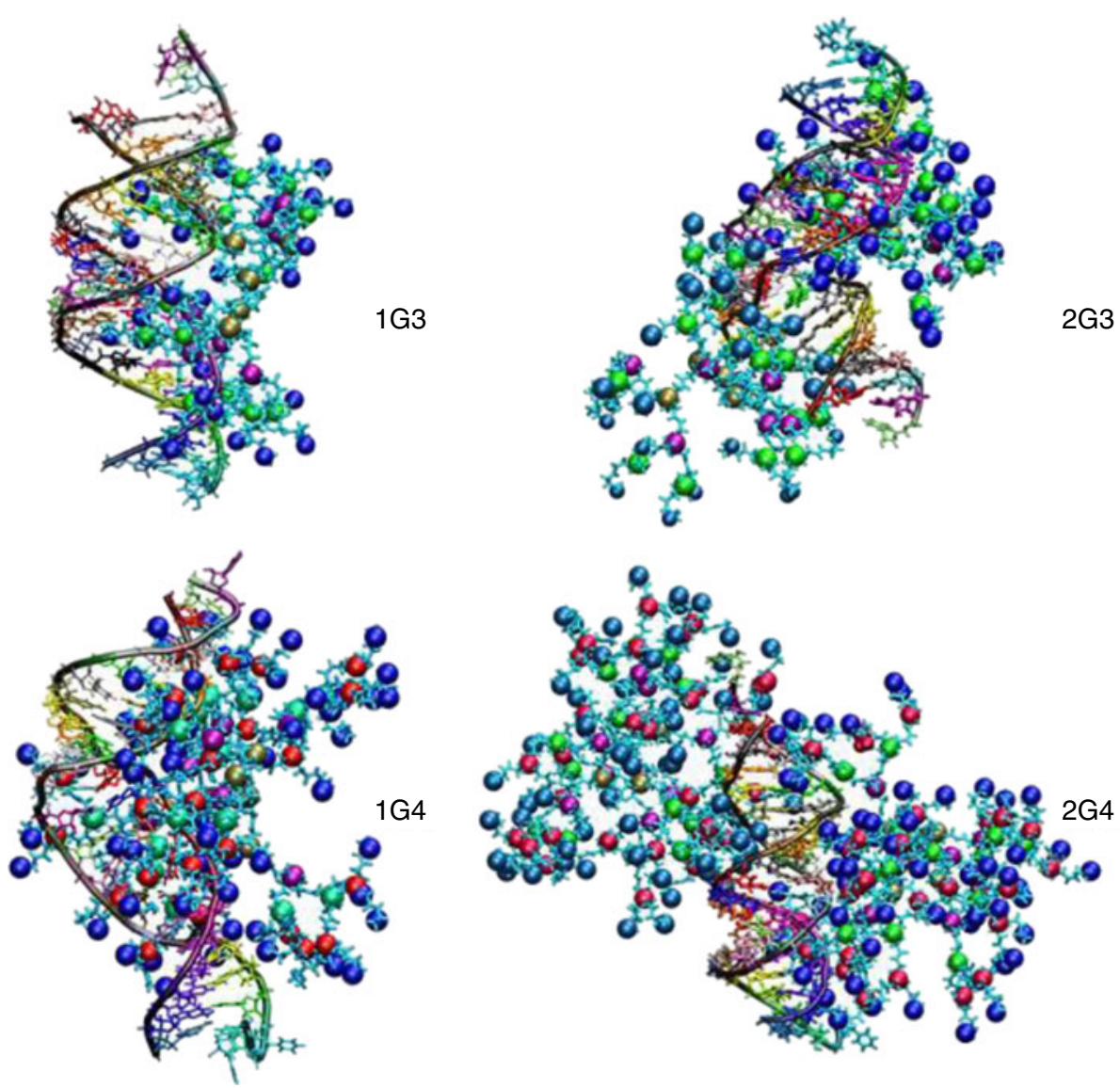

Figure 10. Snapshots of siRNA-dendrimer complexes: siRNA with one dendrimer of generation 3 (1G3), two dendrimers of generation 3 $(2 \mathrm{G} 3)$, one dendrimer of generation $4(1 \mathrm{G} 4)$ and two dendrimers of generation $4(2 \mathrm{G} 4)$. Various amines are levelled different coloured atoms say blue, green, purple and cyan correspond to terminal second, first and core amines in G3, and blue, red, green, purple and cyan correspond to terminal third, second, first and core amines in G4. Figure taken from Vasumathi and Maiti (2010) with permission from the ACS. 


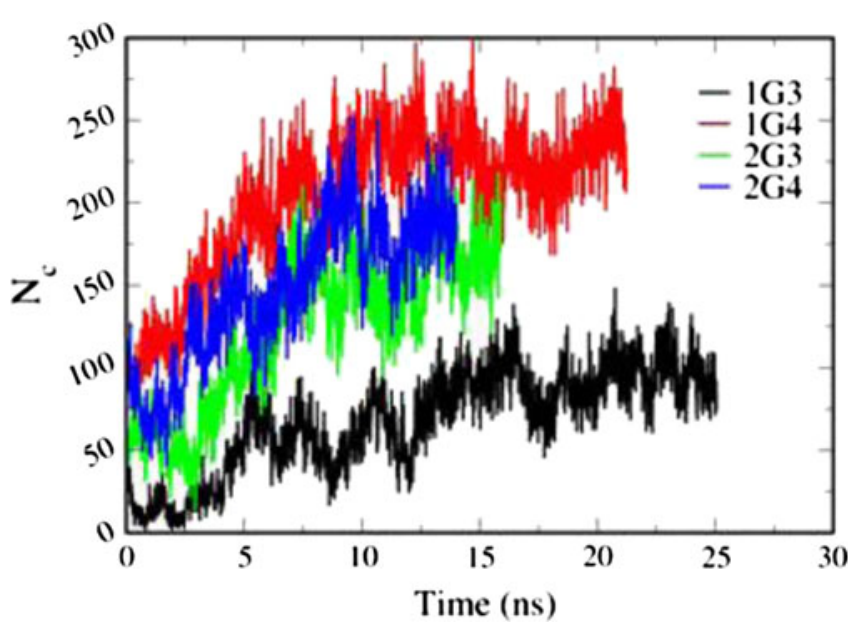

Figure 11. Time evolution of number of close contact atoms between siRNA and dendrimer (any contact within in the hydration shell of siRNA) of generation 3 (1G3) and 4 (1G4) and same generation with two dendrimers (2G3 and $2 \mathrm{G} 4)$. Figure taken from Vasumathi and Maiti (2010) with permission from the ACS.

4.1.5 Binding energy: To understand the thermodynamics of the DNA-dendrimer complexation, we also computed the binding free energy of this complexation using the widely used molecular mechanics Poisson-Boltzmann surface area (MM-PBSA) (Kollman et al. 2000) method. For the entropy calculation, recently developed two-phase (2PT) thermodynamic model (Lin et al. 2003, 2010) was used. The calculated binding energy shows that with the increase in dendrimer generation binding affinity increases and follows the trend G5>G4>G3. Table 1 gives the binding energy of dsDNA complexation with various dendrimer generations.

\subsection{Interaction of siRNA with dendrimer}

4.2.1 siRNA compaction: Although there exist several computational and theoretical studies on the complexation of dendrimers and oppositely charged model polyelectrolytes (Pavan et al. 2009; Lyulin et al. 2005, 2008; Larin et al. 2009a, 2010) and DNA-dendrimer complexation (Maiti and Bagchi 2006; Nandy and Maiti 2011), studies on siRNAdendrimer interaction are really limited. There is a growing interest to study the interaction of siRNA with other nanoscale objects due to their potential application in RNAi therapy. In spite of their current interest, the understanding of binding dynamics between two molecules is not very well established. In 2010, Pavan et al. (2010) reported binding of GL3 siRNA with PAMAM dendrimers of various generations. They concluded that the G4 dendrimer has stronger binding affinity compared to G5 and G6. Later that year, Vasumathi et al. published the interaction between siRNA and different
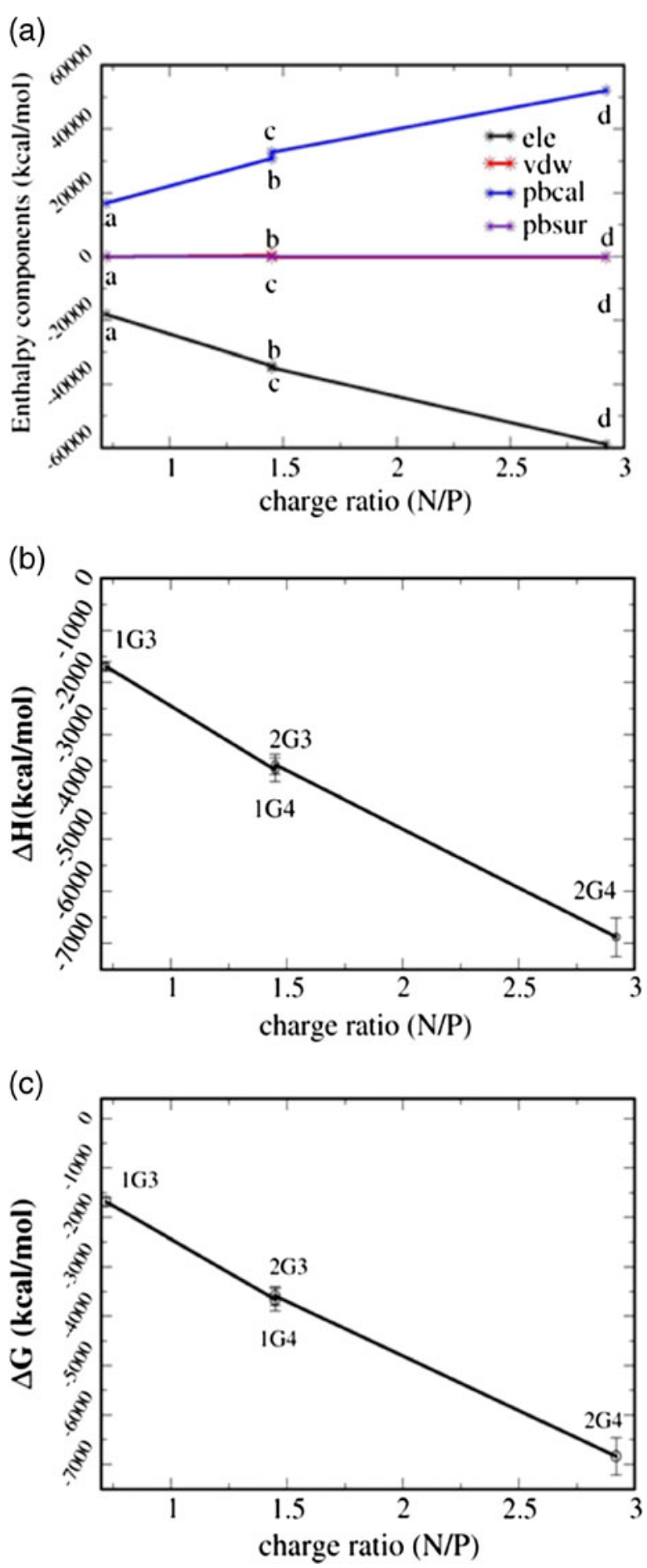

Figure 12. (a) Various enthalpy components with corresponding charge ratios. Here a, b, c and d correspond to $1 \mathrm{G} 3,1 \mathrm{G} 4,2 \mathrm{G} 3$ and $2 \mathrm{G} 4$ complexes respectively. The lines are to guide to eye only. (b) Enthalpy of all the cases with corresponding charge ratios. (c) Binding energies of all the cases with corresponding charge ratios. Figure taken from Vasumathi and Maiti (2010) with permission from the ACS. 
generations of PAMAM dendrimer (Vasumathi and Maiti 2010). They also studied complexation of siRNA and two G3 (2G3) and G4 (2G4) dendrimers separately (figure 10). The key features of this study are highlighted below.

4.2.2 Dynamics of complex formation: Similar to the case of DNA-dendrimer complexation, siRNA also takes first few nanoseconds to start the wrapping and the following few nanoseconds are used to overcome several energetic and entropic hindrances and to find an optimal binding position on the dendrimer surface. One striking feature of siRNA-dendrimer complexation is that the number of contacts between positively charged dendrimer and the negatively charged siRNA does not increase monotonically with increasing number of dendrimers (figure 11). In the case of one dendrimer-siRNA complexation, number of contacts $\left(\mathrm{N}_{\mathrm{c}}\right)$ increased from 1G3 to $1 \mathrm{G} 4$ as the size of $\mathrm{G} 3$ dendrimer is smaller than the size of G4 dendrimer (Maiti and Bagchi 2009; Maiti et al. 2009). Considering the siRNA-2G3 case, the result shows that $\mathrm{N}_{\mathrm{c}}$ for $1 \mathrm{G} 3<\mathrm{N}_{\mathrm{c}}$ for $2 \mathrm{G} 3<\mathrm{N}_{\mathrm{c}}$ for $1 \mathrm{G} 4$. Single $\mathrm{G} 3$ dendrimer cannot wrap the siRNA completely. In the case of $2 \mathrm{G} 3$, dendrimers move away from each other to minimize the repulsion between them and try to wrap the siRNA on both ends of it. Thus, siRNA undergoes a large conformational change and the number of contacts between siRNA and dendrimer increases, and this is more than the case of $1 \mathrm{G} 3$. In contrast, siRNA makes fewer number of contacts with 2G4 dendrimers compared to $1 \mathrm{G} 4$ dendrimer. When $2 \mathrm{G} 4$ dendrimers try to bind the siRNA, none of them are able to wrap the siRNA, and due to inter-dendrimer repulsion, siRNA behaves as a rigid rod. So among all cases, the $2 \mathrm{G} 3$ complex shows better potential to use as a carrier for transfection. One major difference between DNA-dendrimer complexation and siRNA-dendrimer complexation is that during interaction the DNA end-to-end length get shortened and, on the contrary, siRNA end-to-end length increases when it binds to dendrimer. While siRNA interacts with dendrimer, the base pair rise increases, which enables binding to the dendrimer.
4.2.3 Salt concentration: Binding affinity between siRNA and dendrimer decreases as the salt concentration increases. As the salt concentration increases, more and more counterions condense on the surface of the dendrimer, leading to the screening the electrostatic interaction. For $1 \mathrm{G} 3$ and siRNA complex at $10 \mathrm{mM}$ salt concentration, negative charges on siRNA is greatly screened and hence the electrostatic interaction between G3 dendrimer and siRNA is weakened. In contrast, for $1 \mathrm{G} 4$, at the same molarity, because of greater number of positive charges for G4 dendrimer, the electrostatic interaction between siRNA and dendrimer is less affected compared to G3. With the increase in the salt concentration to $150 \mathrm{mM}$, the binding affinity for G4 is also greatly reduced because of strong screening of electrostatic interaction.

4.2.4 Binding energy: Binding free energy analysis depicts some interesting features of the siRNA-dendrimer complex. MMPB-SA and 2PT methods were used to calculate the binding enthalpy and entropy respectively. Charge ratio, N/P, increases when dendrimer generation goes from lower (G3) to higher (G4) generation. For a particular generation, by increasing the number of dendrimers (1G3 to $2 \mathrm{G} 3$ ) also, one can increase the N/P ratio. This implies that the binding affinity between siRNA and 1G3/1G4 is stronger than siRNA-2G3/ $2 \mathrm{G} 4$. Analysing the enthalpy components reveal some important features (figure 12a-b). Increasing charge ratio does not have much influence on the vdW component and the surface area contribution (PBSURF). In contrast, there is a positive increment of solvent electrostatic energy (PBCAL) with increasing generation and concentration of dendrimer. In the same condition, electrostatic energy of the gas phase (solute) increases negatively. Hence, the major contribution of the electrostatic interaction during binding of siRNA dendrimer is clearly evident from the above analysis, and the reason behind this interaction is the attraction between positively charged groups of protonated amines on the surface of dendrimer and the negatively charges phosphate on the backbone of the DNA, respectively. Figure $12 \mathrm{~b}$ and $\mathrm{c}$ presents the enthalpy of binding and total binding free

Table 2. Binding free energy using MM-PB/GB SA and $2 \mathrm{PT}$ methods for siRNA-dendrimer complex

\begin{tabular}{lccr}
\hline Complex & $\Delta \mathrm{H}(\mathrm{kcal} / \mathrm{mol})$ & $-\mathrm{T} \Delta \mathrm{S}(\mathrm{kcal} / \mathrm{mol})$ & $\Delta \mathrm{G}(\mathrm{kcal} / \mathrm{mol})$ \\
\hline $1 \mathrm{G} 3$ & $-1689.84 \pm 92.54$ & 5.58 & $-1684.26 \pm 92.54$ \\
$1 \mathrm{G} 4$ & $-3672.99 \pm 225.05$ & 9.35 & $-3663.64 \pm 225.05$ \\
$2 \mathrm{G} 3$ & $-3570.95 \pm 191.76$ & -19.41 & $-3590.36 \pm 191.76$ \\
$2 \mathrm{G} 4$ & $-6880.43 \pm 373.4$ & 39.8 & $-6840.63 \pm 373.4$ \\
$1 \mathrm{G} 3-10 \mathrm{mM}$ & $-479.5 \pm 28.53$ & 11.52 & $-467.98 \pm 28.53$ \\
$1 \mathrm{G} 3-150 \mathrm{mM}$ & $-198.43 \pm 7.68$ & 3.11 & $-195.32 \pm 7.68$ \\
$1 \mathrm{G} 4-10 \mathrm{mM}$ & $-3465.36 \pm 192.27$ & 9.02 & $-3456.34 \pm 192.27$ \\
$1 \mathrm{G} 4-150 \mathrm{mM}$ & $-2206.73 \pm 110.19$ & 8.24 & $-2198.49 \pm 110.19$ \\
\hline
\end{tabular}

Table taken from Vasumathi and Maiti (2010) with permission from the ACS. 
energy as a function of charge ratio of the complex. We see that the both binding enthalpy and free energy increase with increase in the charge ratio of siRNA and dendrimer. Table 2 gives the binding energy for various dendrimer generations complexed with siRNA.

\section{Summary and outlook}

To summarize, we have given a molecular-level picture of nucleic acid interaction and compaction by variety of nanoscale objects such as CNTs, graphene and dendrimers. Apart from their application in gene therapy and RNAi, such interactions offer a route to designing various functional nanostructures. In particular, we studied the stability of dsDNA in the presence of CNTs. Structural transitions from A-to-B form in physiological conditions in the presence and absence of CNT was studied. In the presence of CNT, the transition from A-to-B form becomes slower compared to the case when dsDNA is in bulk solution. The van der Waals interaction between A-DNA and CNT makes the transition a little slower. This transition does not happen when $80 \%$ ethanol is added to the solution. This is due to the lower dielectric constant of ethanol and water mixture compared to water, and this stabilizes A-form DNA over B-DNA. When $\mathrm{B}-\mathrm{DNA}$ is in the presence of CNT/graphene, the vdW interaction helps initiate unzipping of few base pairs in dsDNA, which facilitates further binding and adsorption on CNT/ graphene. However, we do not see complete wrapping of dsDNA on the CNT surface. The unzipping and adsorption of dsDNA on graphene is larger compared to the case of CNT. The ssDNA wraps around CNT very well compared to the dsDNA. We have also demonstrated that ssDNA enters the CNT partially but does not translocate fully. In contrast, dsDNA does not enter the interior of the CNT. Whether this is an artefact of the force field used or the kinetics is too slow to give rise to the entry in the simulation time scale has not yet been explored. In future, we plan to study the translocation under the influence of electric field gradient along the CNT axis. Apart from their application in gene therapy and RNAi, understanding DNA-CNT and DNA-graphene hybrid structures is important in CNT separation, DNA sequencing and sensing within living cells (Zheng et al. 2003a, b; Chou et al. 2004; Heller et al. 2006; Zhao and Johnson 2007; Johnson et al. 2008). In the case of dendrimer interaction with DNA and siRNA, we highlighted various advantageous properties of the PAMAM dendrimer. It has been demonstrated that the topology and the unique $\mathrm{pH}$ dependency of the dendrimer has the attributes that make them an attractive carrier for gene therapy. Studies on interaction between PAMAM and DNA/siRNA are promising and show that this dendrimer is very efficient and less toxic compared to other carriers. By controlling dendrimer size and conformation by varying generation number, one can fine-tune the pharmacokinetic properties of the dendrimer. Thus, PAMAM has significant advantages compared to other linear polymers. We have shown atomistic details of the interaction between PAMAM dendrimers of varying generations and DNA (both ssDNA and dsDNA)/siRNA. How the nature of the interaction changes with varying $\mathrm{pH}$ and as a function of dendrimer generation and concentration of dendrimer was discussed in detail in the present article. Recent advancement in this field shows a very promising future in biomedical applications of this molecule.

\section{Acknowledgements}

We thank the Department of Biotechnology, India, for financial support.

\section{References}

Bergstrom CT and Antiat R 2005 On RNA interference as template immunity. J. Biosci. 30 295-297

Bielinska AU, KukowskaLatallo JF and Baker JR 1997 The interaction of plasmid DNA with polyamidoamine dendrimers: mechanism of complex formation and analysis of alterations induced in nuclease sensitivity and transcriptional activity of the complexed DNA. Biochim. Biophys. Acta - Gene Struc. Exp. 1353 180-190

Bloomfield VA, Crothers DM and Tinoco I (2000) Nucleic acids: structures, properties, and functions (California, University Science Books)

Bosman AW, Janssen HM and Meijer EW 1999 About dendrimers: Structure, physical properties, and applications. Chem. Rev. 99 $1665-1688$

Brooks BR, Brooks CL, Mackerell AD, Nilsson L, Petrella RJ, Roux B, Won Y, Archontis G, et al. 2009 CHARMM: The Biomolecular Simulation Program. J. Comput. Chem. 30 $1545-1614$

Calladine CR and Drew HR 1984 A Base-Centered Explanation of the B-to-a Transition in DNA. J. Mol. Biol. 178 773-781

Case DA, Darden TA, Cheatham TE, et al. 2006 AMBER9 (San Francisco: University of California)

Castro Neto AH, Guinea F, Peres NMR, Novoselov KS and Geim AK 2009 The electronic properties of graphene. Rev. Modern Phy. 81 109-162

Cheatham TE and Kollman PA 1996 Observation of the A-DNA to B-DNA transition during unrestrained molecular dynamics in aqueous solution. J. Mol. Biol. 259 434-444

Chou SG, Ribeiro HB, Barros EB, Santos AP, Nezich D, Samsonidze GG, Fantini C, Pimenta MA, et al. 2004 Optical characterization of DNA-wrapped carbon nanotube hybrids. Chem. Phy. Lett. 397 296-301

Dickerson RE 1992 DNA-Structure from a to Z. Methods Enzymol. $21167-111$

Duan Y, Wu C, Chowdhury S, Lee MC, Xiong GM, Zhang W, Yang R, Cieplak P, et al. 2003 A point-charge force field for molecular mechanics simulations of proteins based on 
condensed-phase quantum mechanical calculations. J. Comput. Chem. 24 1999-2012

Dufes C, Uchegbu IF and Schatzlein AG 2005 Dendrimers in gene delivery. Adv. Drug Delivery Rev. 57 2177-2202

Duxbury MS, Ito H, Benoit E, Zinner MJ, Ashley SW and Whang EE 2003 RNA interference targeting focal adhesion kinase enhances pancreatic adenocarcinoma gemcitabine chemosensitivity. Biochem. Biophys. Res. Commun. 311 786-792

Elbashir SM, Harborth J, Lendeckel W, Yalcin A, Weber K and Tuschl T 2001a Duplexes of 21-nucleotide RNAs mediate RNA interference in cultured mammalian cells. Nature 411 494-498

Elbashir SM, Lendeckel W and Tuschl T 2001b RNA interference is mediated by 21-and 22-nucleotide RNAs. Genes Dev. 15188 200

Fant K, Esbjorner EK, Lincoln P and Norden B 2008 DNA condensation by PAMAM dendrimers: Self-assembly characteristics and effect on transcription. Biochemistry 47 1732-1740

Fire A, Xu SQ, Montgomery MK, Kostas SA, Driver SE and Mello CC 1998 Potent and specific genetic interference by doublestranded RNA in Caenorhabditis elegans. Nature 391 806-811

Franklin RE and Gosling RG 1953 Molecular configuration in sodium thymonucleate. Nature 171 740-741

Frechet JMJ 1994 Functional polymers and dendrimers: reactivity, molecular architecture, and interfacial energy. Science $\mathbf{2 6 3}$ $1710-1715$

Gao HJ, Kong Y, Cui DX and Ozkan CS 2003 Spontaneous insertion of DNA oligonucleotides into carbon nanotubes. Nano Lett. 3 471-473

Garaj S, Hubbard W, Reina A, Kong J, Branton D and Golovchenko JA 2010 Graphene as a subnanometre transelectrode membrane. Nature 467 190-193

Geim AK and Novoselov KS 2007 The rise of graphene. Nat. Mater. 6 183-191

Grayson SM and Frechet JMJ 2001 Convergent dendrons and dendrimers: from synthesis to applications. Chem. Rev. 101 3819-3867

Grayson ACR, Doody AM and Putnam D 2006 Biophysical and structural characterization of polyethylenimine-mediated siRNA delivery in vitro. Pharmaceut. Res. 23 1868-1876

Haensler J and Szoka FC 1993 Polyamidoamine cascade polymers mediate efficient transfection of cells in culture. Bioconjugate Chem. 4 372-379

Hannon GJ 2002 RNA interference. Nature 418 244-251

Hannon GJ and Rossi JJ 2004 Unlocking the potential of the human genome with RNA interference. Nature 431 371-378

Harries D, May S, Gelbart WM and Ben-Shaul A 1998 Structure, stability, and thermodynamics of lamellar DNA-lipid complexes. Biophys. J. 75 159-173

Heller DA, Jeng ES, Yeung TK, Martinez BM, Moll AE, Gastala JB and Strano MS 2006 Optical detection of DNA conformational polymorphism on single-walled carbon nanotubes. Science $\mathbf{3 1 1} 508-511$

Herskovits T and Singer SJ 1961 Nonaqueous solutions of DNA denaturation in methanol and ethanol. Arch. Biochem. Biophy. 94 99-114

Ivanov VI, Minchenkova LE, Minyat EE, Frank-Kamenetskii MD and Schyolkina AK 1974 The B to A transition of DNA in solution. J. Mol. Biol. 87 817-833
Jacobomolina A, Ding JP, Nanni RG, Clark AD, Lu XD, Tantillo C, Williams RL, Kamer G, et al. 1993 Crystal-structure of human-immunodeficiency-virus type-1 reverse-transcriptase complexed with double-stranded DNA at 3.0 angstrom resolution shows bent DNA. Proc. Nat. Acad. Sci. USA 90 6320-6324

Jacque JM, Triques K and Stevenson M 2002 Modulation of HIV-1 replication by RNA interference. Nature 418 435-438

Jayaram B, Sprous D, Young MA and Beveridge DL 1998 Free energy analysis of the conformational preferences of A and B forms of DNA in solution. J. Am. Chem. Soc. 12010629 10633

Johnson RR, Johnson ATC and Klein ML 2008 Probing the structure of DNA-carbon nanotube hybrids with molecular dynamics. Nano Lett. 8 69-75

Jones S, van Heyningen P, Berman HM and Thornton JM 1999 Protein-DNA interactions: A structural analysis. J. Mol. Biol. 287 877-896

Jovin TM, Soumpasis DM and McIntosh LP 1987 The Transition between B-DNA and Z-DNA. Annu. Rev. Phys. Chem. 38 521-560

Kiefer JR, Mao C, Braman JC and Beese LS 1998 Visualizing DNA replication in a catalytically active Bacillus DNA polymerase crystal. Nature 391 304-307

Kim DH and Rossi JJ 2007 Strategies for silencing human disease using RNA interference. Nat. Rev. Genet. 8 173-184

Klug A 2004 The discovery of the DNA double helix. J. Mol. Biol. 335 3-26

Kollman PA, Massova I, Reyes C, Kuhn B, Huo SH, Chong L, Lee M, Lee T, et al. 2000 Calculating structures and free energies of complex molecules: Combining molecular mechanics and continuum models. Acc. Chem. Res. 33 889-897

KukowskaLatallo JF, Bielinska AU, Johnson J, Spindler R, Tomalia DA and Baker JR 1996 Efficient transfer of genetic material into mammalian cells using Starburst polyamidoamine dendrimers. Proc. Nat. Acad. Sci. USA 93 4897-4902

Kurreck J 2009 RNA Interference: From basic research to therapeutic applications. Angewandte Chemie-Intl. Ed. 48 1378-1398

Larin S, Lyulin S, Lyulin A and Darinskii A 2009a Computer Simulations of Interpolyelectrolyte Complexes Formed by Star-like Polymers and Linear Polyelectrolytes. Macromolecular Symposia 278 40-47

Larin SV, Lyulin SV, Lyulin AV and Darinskii AA 2009b Charge inversion of dendrimers in complexes with linear polyelectrolytes in the solutions with low pH. Polymer Sci. Ser. A 51459 468

Larin SV, Darinskii AA, Lyulin AV and Lyulin SV 2010 Linker formation in an overcharged complex of two dendrimers and linear polyelectrolyte. J. Phys. Chem. B 114 2910-2919

Lee NS, Dohjima T, Bauer G, Li HT, Li MJ, Ehsani A, Salvaterra P and Rossi J 2002 Expression of small interfering RNAs targeted against HIV-1 rev transcripts in human cells. Nat. Biotechnol. 20 500-505

Lin ST, Blanco M and Goddard WA 2003 The two-phase model for calculating thermodynamic properties of liquids from molecular dynamics: Validation for the phase diagram of Lennard-Jones fluids. J. Chem. Phys. 119 11792-11805

Lin ST, Maiti PK and Goddard WA 2010 Two-phase thermodynamic model for efficient and accurate absolute entropy of water 
from molecular dynamics simulations. J. Phys. Chem. B 114 8191

Liu Z, Winters M, Holodniy M and Dai HJ 2007 siRNA delivery into human $\mathrm{T}$ cells and primary cells with carbon-nanotube transporters. Angewandte Chemie-Intl. Ed. 46 2023-2027

Liu Z, Chen K, Davis C, Sherlock S, Cao QZ, Chen XY and Dai HJ 2008 Drug delivery with carbon nanotubes for in vivo cancer treatment. Cancer Res. 68 6652-6660

Liu Z, Tabakman S, Welsher K and Dai HJ 2009 Carbon nanotubes in biology and medicine: in vitro and in vivo detection, imaging and drug delivery. Nano Res. 2 85-120

Livolant F 1991 Ordered phases of DNA in vivo and in vitro. Physica A 176 117-137

Lu XJ, Shakked Z and Olson WK 2000 A-form conformational motifs in ligand-bound DNA structures. J. Mol. Biol. 300 819-840

Lu Q, Moore JM, Huang G, Mount AS, Rao AM, Larcom LL and Ke PC 2004 RNA polymer translocation with single-walled carbon nanotubes. Nano Lett. 4 2473-2477

Lyulin SV, Darinskii AA and Lyulin AV 2005 Computer simulation of complexes of dendrimers with linear polyelectrolytes. Macromolecules 38 3990-3998

Lyulin SV, Vattulainen I and Gurtovenko AA 2008 Complexes comprised of charged dendrimers, linear polyelectrolytes, and counterions: Insight through coarse-grained molecular dynamics simulations. Macromolecules 41 4961-4968

Maiti PK and Bagchi B 2006 Structure and dynamics of DNAdendrimer complexation: Role of counterions, water, and base pair sequence. Nano Lett. 6 2478-2485

Maiti PK and Bagchi B 2009 Diffusion of flexible, charged, nanoscopic molecules in solution: Size and $\mathrm{pH}$ dependence for PAMAM dendrimer. J. Chem. Phys. 131214901

Maiti PK, Cagin T, Wang GF and Goddard WA 2004 Structure of PAMAM dendrimers: Generations 1 through 11. Macromolecules 37 6236-6254

Maiti PK, Li YY, Cagin T and Goddard WA 2009 Structure of polyamidoamide dendrimers up to limiting generations: A mesoscale description. J. Chem. Phys. 130144902

Meister G and Tuschl T 2004 Mechanisms of gene silencing by double-stranded RNA. Nature 431 343-349

Merchant CA, Healy K, Wanunu M, Ray V, Peterman N, Bartel J, Fischbein MD, Venta K, et al. 2010 DNA Translocation through Graphene Nanopores. Nano Lett. 10 2915-2921

Miller JL and Kollman PA 1997 Observation of an A-DNA to B-DNA transition in a nonhelical nucleic acid hairpin molecule using molecular dynamics. Biophys. J. 73 2702-2710

Mintzer MA and Simanek EE 2009 Nonviral Vectors for gene delivery. Chem. Rev. 109 259-302

Nandy B and Maiti PK 2011 DNA compaction by a dendrimer. $J$. Phys. Chem. B 115 217-230

Napoli C, Lemieux C and Jorgensen R 1990 Introduction of a chimeric chalcone synthase gene into petunia results in reversible co-suppression of homologous genes in trans. Plant Cell 2 279-289

Novoselov KS, Geim AK, Morozov SV, Jiang D, Zhang Y, Dubonos SV, Grigorieva IV and Firsov AA 2004 Electric field effect in atomically thin carbon films. Science 306 666-669

Ottaviani MF, Furini F, Casini A, Turro NJ, Jockusch S, Tomalia DA and Messori L 2000 Formation of supramolecular structures between DNA and starburst dendrimers studied by EPR, CD, UV, and melting profiles. Macromolecules 33 7842-7851

Pavan GM, Danani A, Pricl S and Smith DK 2009 Modeling the multivalent recognition between dendritic molecules and DNA: Understanding how ligand "sacrifice" and screening can enhance binding. J. Am. Chem. Soc. 131 9686-9694

Pavan GM, Albertazzi L and Danani A 2010 Ability to adapt: different generations of PAMAM dendrimers show different behaviors in binding siRNA. J. Phys. Chem. B 114 2667-2675

Reynolds JA and Hough JM 1957 Formulae for dielectric constant of mixtures. Proc. Phys. Soc. London Sec. B 70 769-775

Rich A and Zhang SG 2003 Z-DNA: the long road to biological function. Nat. Rev. Genet. 4 566-572

Saenger W (1984) Principles of nucleic acid structure (New York: Springer-Verlag)

Santosh M and Maiti PK 2009 Force induced DNA melting. J. Phys. - Condensed Matter 21034113

Santosh M and Maiti PK 2011 Structural rigidity of paranemic crossover and juxtapose DNA nanostructures. Biophys. J. 101 1393-1402

Santosh M, Panigrahi S, Bhattacharyya D, Sood AK and Maiti PK 2012 Unzipping and binding of small interfering RNA with single walled Carbon Nanotube: a platform for small interfering RNA delivery. J. Chem. Phys.136 065106

Scherrenberg R, Coussens B, van Vliet P, Edouard G, Brackman J, de Brabander E and Mortensen K 1998 The molecular characteristics of poly(propyleneimine) dendrimers as studied with smallangle neutron scattering, viscosimetry, and molecular dynamics. Macromolecules 31 456-461

Schiessel H 2003 The physics of chromatin. J. Phys. - Condensed Matter 15 R699-R774

Schneider GF, Kowalczyk SW, Calado VE, Pandraud G, Zandbergen HW, Vandersypen LMK and Dekker C 2010 DNA Translocation through graphene nanopores. Nano Lett. 10 3163-3167

Soutschek J, Akinc A, Bramlage B, Charisse K, Constien R, Donoghue M, Elbashir S, Geick A, et al. 2004 Therapeutic silencing of an endogenous gene by systemic administration of modified siRNAs. Nature 432 173-178

Sponer J, Jurecka P and Hobza P 2004 Accurate interaction energies of hydrogen-bonded nucleic acid base pairs. J. Am. Chem. Soc. 126 10142-10151

Stankovich S, Dikin DA, Dommett GHB, Kohlhaas KM, Zimney EJ, Stach EA, Piner RD, Nguyen ST and Ruoff RS 2006 Graphene-based composite materials. Nature $\mathbf{4 4 2}$ 282-286

Tsubouchi A, Sakakura J, Yagi R, Mazaki Y, Schaefer E, Yano H and Sabe H 2002 Localized suppression of RhoA activity by Tyr31/118-phosphorylated paxillin in cell adhesion and migration. J. Cell Biol. 159 673-683

Urban-Klein B, Werth S, Abuharbeid S, Czubayko F and Aigner A 2005 RNAi-mediated gene-targeting through systemic application of polyethylenimine (PEI)-complexed siRNA in vivo. Gene Ther. 12 461-466

Vangunsteren WF and Berendsen HJC 1987 GROMOS-87 Manual.

Vasumathi V and Maiti PK 2010 Complexation of siRNA with dendrimer: A molecular modeling approach. Macromolecules 43 8264-8274 
Watson JD and Crick FHC 1953 Molecular Structure of nucleic acids - a structure for deoxyribose nucleic acid. Nature 171 737-738

Welch P and Muthukumar M 2000 Dendrimer-polyelectrolyte complexation: A model guest-host system. Macromolecules 33 6159-6167

Wilkins MHF, Stokes AR and Wilson HR 1953 Molecular Structure of Deoxypentose Nucleic Acids. Nature 171 738-740

Zamore PD and Aronin N 2003 siRNAs knock down hepatitis. Nat. Med. 9 266-267

Zhang YB, Tan YW, Stormer HL and Kim P 2005 Experimental observation of the quantum Hall effect and Berry's phase in graphene. Nature 438 201-204

Zhao X and Johnson JK 2007 Simulation of adsorption of DNA on carbon nanotubes. J. Am. Chem. Soc. 129 10438-10445

Zheng M, Jagota A, Semke ED, Diner BA, McLean RS, Lustig SR, Richardson RE and Tassi NG 2003a DNA-assisted dispersion and separation of carbon nanotubes. Nat. Mater. 2 338-342

Zheng M, Jagota A, Strano MS, Santos AP, Barone P, Chou SG, Diner BA, Dresselhaus MS, et al. 2003b Structure-based carbon nanotube sorting by sequence-dependent DNA assembly. Science 302 1545-1548

Zimmerman SB and Pheiffer BH 1979 Direct demonstration that the ethanol-induced transition of DNA is between the a-forms and B-forms - X-ray-diffraction study. J. Mol. Biol. 135 10231027

Zimmerman SC, Zeng FW, Reichert DEC and Kolotuchin SV 1996 Self-assembling dendrimers. Science 271 1095-1098

Zinchenko AA and Chen N 2006 Compaction of DNA on nanoscale three-dimensional templates. J. Phys. - Condensed Matter 18 R453-R480 